\title{
Thermal 2-loop master spectral function at finite momentum
}

\section{Laine}

Institute for Theoretical Physics (ITP),

Albert Einstein Center for Fundamental Physics (AEC), University of Bern,

Sidlerstrasse 5, CH-3012 Bern, Switzerland

E-mail: laine@itp.unibe.ch

ABSTRACT: When considering NLO corrections to thermal particle production in the "relativistic" regime, in which the invariant mass squared of the produced particle is $\mathcal{K}^{2} \sim(\pi T)^{2}$, then the production rate can be expressed as a sum of a few universal "master" spectral functions. Taking the most complicated 2-loop master as an example, a general strategy for obtaining a convergent 2-dimensional integral representation is suggested. The analysis applies both to bosonic and fermionic statistics, and shows that for this master the nonrelativistic approximation is only accurate for $\mathcal{K}^{2} \gtrsim(8 \pi T)^{2}$, whereas the zero-momentum approximation works surprisingly well. Once the simpler masters have been similarly resolved, NLO results for quantities such as the right-handed neutrino production rate from a Standard Model plasma or the dilepton production rate from a QCD plasma can be assembled for $\mathcal{K}^{2} \sim(\pi T)^{2}$.

KeYwords: Thermal Field Theory, Quark-Gluon Plasma, Neutrino Physics

ARXIV EPRINT: 1304.0202 


\section{Contents}

1 Introduction 1

2 Physical observables $\quad 4$

$\begin{array}{lll}3 & \text { Splitup into real and virtual corrections } & 7\end{array}$

4 Real corrections $\quad 8$

4.1 Integration variables 8

$\begin{array}{lll}4.2 & \text { Crossing symmetry } & 12\end{array}$

$\begin{array}{lll}4.3 & \text { Inner integrations } & 13\end{array}$

5 Virtual corrections $\quad 15$

$\begin{array}{lll}6 & \text { Cancellation of divergences } & 17\end{array}$

$\begin{array}{llr}7 & \text { Final result } & 20\end{array}$

8 Numerical evaluation $\quad 22$

$\begin{array}{lll}9 & \text { Summary and outlook } & 23\end{array}$

\section{Introduction}

Given the remarkable confirmation of microscopic Standard Model and QCD physics through the Large Hadron Collider program, it appears well-motivated to work out how the same interactions behaved in the macroscopic thermal environment of the Early Universe. Whereas there are good tools available for doing this for thermodynamic quantities such as the overall equation of state, much less is known about real-time rates, such as particle production rates or the equilibration rates related to the most weakly interacting degrees of freedom. Indeed, in most cases only phenomenological estimates or leading-order (LO) weak-coupling expressions are available. Given the known infrared problems of thermal field theory, which imply that next-to-leading order (NLO) corrections may be surprisingly large, further work is needed in order to get an impression on the numerical accuracy of the results currently available.

In relativistic thermal field theory, the structure of the weak-coupling expansion depends sensitively on the physical scales of the problem. Focussing on the simplest situation, in which there is only one zero-temperature mass scale, denoted by the invariant mass squared $\mathcal{K}^{2}>0$, there are different "regimes" depending on the ratio $\mathcal{K}^{2} /(\pi T)^{2}{ }^{1}$ In the

\footnotetext{
${ }^{1}$ To be precise, because of the absence of Lorentz invariance within a heat bath the magnitudes of $k_{ \pm} \equiv\left(k_{0} \pm k\right) / 2$ need to be separately compared with $\pi T$, and the number of regimes may proliferate.
} 


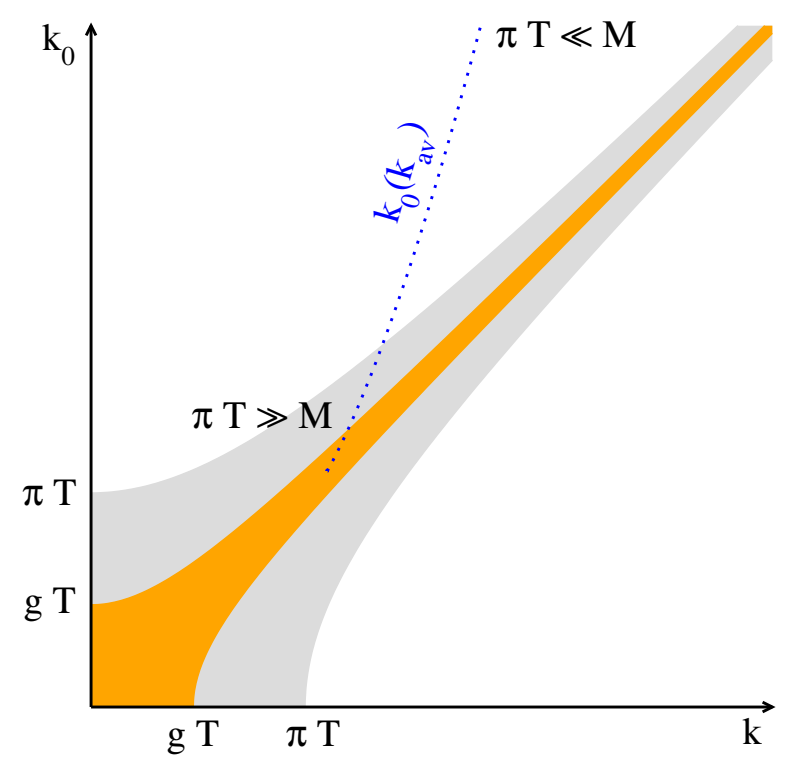

Figure 1. An example of how during the cosmological evolution, different kinematic regimes are crossed in the thermal production of a particle species with a fixed invariant mass $\mathcal{K}^{2} \equiv M^{2}$. A generic weak gauge coupling is denoted by $g\left[\alpha \equiv g^{2} /(4 \pi)\right]$, and $k_{\text {av }}$ is defined by eq. (8.3). If a fixed invariant energy $\left(E_{\mu^{-}}+E_{\mu^{+}}\right)^{2}-\left(\mathbf{k}_{\mu^{-}}+\mathbf{k}_{\mu^{+}}\right)^{2} \simeq 1 \mathrm{GeV}^{2}$ of a dilepton pair is considered, a similar evolution takes place during a heavy ion collision, however the temperature range is rather narrow.

so-called non-relativistic regime, $\mathcal{K}^{2} \gg(\pi T)^{2}$, results can be represented in the form of an Operator Product Expansion [1], and thermal corrections are in general small (powersuppressed). In contrast, in the so-called ultrarelativistic regime, $\mathcal{K}^{2} \ll(\pi T)^{2}$, the naive loop expansion breaks down, and extensive resummations (incorporating for instance the physics of the Landau-Pomeranchuk-Migdal effect) are needed for determining even the LO result. The current technology for this has been developed in the context of the photon production rate from a QCD plasma $[2-4]^{2}$ and subsequently reformulated and applied to the right-handed neutrino production rate from a Standard Model plasma [8-10]. (Very recently the photon production rate has been determined up to NLO [11], which here means $\mathcal{O}\left(\alpha^{1 / 2}\right)$ rather than the usual $\mathcal{O}(\alpha)$, where $\alpha \equiv g^{2} /(4 \pi)$.)

The focus of the present paper is the so-called relativistic regime, $\mathcal{K}^{2} \sim(\pi T)^{2}$. Such results may permit for an interpolation between the non-relativistic and ultrarelativistic cases, thereby perhaps yielding phenomenologically broadly applicable expressions (the cosmological evolution is illustrated in figure 1). At NLO, which in the relativistic regime means $\mathcal{O}(\alpha)$, no infrared divergences are expected to be encountered in the full result, and there is no need for resummations either. However, because of the loss of Lorentz symmetry within a thermal bath, the analysis is technically cumbersome. This is the case particularly if a non-zero momentum $k \equiv|\mathbf{k}| \neq 0$ is considered, in which case even LO expressions are non-trivial [12]. Nevertheless, as will be demonstrated, NLO results can be worked out with some effort.

\footnotetext{
${ }^{2}$ Similar resummations are needed for dilepton production in the soft regime $\mathcal{K}^{2} \ll(\pi T)^{2}[5-7]$.
} 
Examples of concrete applications that we have in mind are the right-handed neutrino production rate in the Early Universe, motivated by leptogenesis or dark matter computations $[13,14]$, and the dilepton production rate in hot QCD, motivated by heavy ion collision experiments. For the former, the NLO level has already been reached in the non-relativistic regime $[15,16]$. For the latter, the NLO level was reached long ago at vanishing momentum in the relativistic regime [17-19]. More recently, the case of a vanishing momentum in the relativistic regime has been studied more generally [20], expressing results in terms of a finite number of universal "master" structures; in particle language the cases considered were the (off-shell) thermal axion and dilaton [20] or graviton [21] production rates. (The physical relevance of these computations is related to lattice determinations of the corresponding "transport coefficients", which are the rate of anomalous chirality violation and the bulk and shear viscosities. Indeed, because of the necessity of analytic continuation from Euclidean signature, ultraviolet features need to subtracted from non-perturbative data before an extrapolation to $k_{0} \rightarrow 0$ becomes possible even in principle [22-24].)

The purpose of the present paper is to evaluate the spectral function corresponding to the most complicated 2-loop master topology (figure 2) at non-zero momentum in the relativistic regime. This topology has merited extensive investigations under other circumstances. Indeed, at zero temperature in 3 space-time dimensions even the massive case can be solved [25], whereas in the physical 4-dimensional case the result has an extremely rich structure [26] which, despite a vast body of work, still remains under further investigation today (see, e.g., ref. [27] and references therein). In the massless limit, however, the result vanishes at zero temperature. Nevertheless the spectral function (cut) is non-zero and possesses a rich structure at finite temperature. Previous thermal analyses exist at zero momentum [20] as well as at zero energy but non-zero momentum [28], the latter case corresponding to the space-like domain relevant for the physics of plasma screening.

Recently, a separate line of study of thermal particle production in the relativistic regime has been initiated whose goals appear to partly overlap with those of the present paper [29]. The authors give a 4-dimensional integral representation for the gauge boson contribution to the right-handed neutrino production rate, but no numerical evaluation is shown. Given the technical complexity of the problem, it appears welcome that two independent and methodologically different computations are being pursued, permitting in the end to crosscheck the validity of both results.

The plan of this paper is the following. We start by recalling how NLO results for two physical observables can be expressed in terms of a finite number of simple master sumintegrals (section 2). After carrying out Matsubara sums, the cut of the most complicated master can furthermore be decomposed into processes representing real and virtual corrections (section 3). The real corrections are analyzed in section 4, showing that through a suitable choice of variables they can be given a 2-dimensional integral representation (soft and collinear divergences are regulated by an auxiliary mass parameter at this stage); the same task is accomplished for the virtual corrections in section 5. Both real and virtual corrections are divergent if the auxiliary mass parameter is sent to zero; in section 6 it is shown that the sum remains finite. Finally a form suitable for practical evaluation is given in section 7, whereas numerical comparisons with known limiting values comprise section 8 . A brief summary and outlook is offered in section 9 . 


\section{Physical observables}

To underline the significance of the master spectral function considered, we start by recalling two separate physics contexts in which it plays a role.

To leading order in $\alpha_{e m} \equiv e^{2} /(4 \pi)$, the production rate of $\mu^{-} \mu^{+}$pairs from a hot QCD medium can be expressed as [30-32]

$$
\frac{\mathrm{d} N_{\mu^{-} \mu^{+}}}{\mathrm{d}^{4} \mathcal{X} \mathrm{d}^{4} \mathcal{K}}=\sum_{q q^{\prime}} \frac{-2 e^{4} Q_{q} Q_{q^{\prime}} \theta\left(\mathcal{K}^{2}-4 m_{\mu}^{2}\right)}{3(2 \pi)^{5} \mathcal{K}^{2}}\left(1+\frac{2 m_{\mu}^{2}}{\mathcal{K}^{2}}\right)\left(1-\frac{4 m_{\mu}^{2}}{\mathcal{K}^{2}}\right)^{\frac{1}{2}} n_{\mathrm{B}}\left(k_{0}\right) \rho_{q q^{\prime}}(\mathcal{K}),
$$

where $Q_{q}$ is the electric charge of quark of flavour $q$ in units of $e, n_{\mathrm{B}}$ is the Bose distribution, and

$$
\rho_{q q^{\prime}}(\mathcal{K}) \equiv \int_{\mathcal{X}} e^{i \mathcal{K} \cdot \mathcal{X}}\left\langle\frac{1}{2}\left[\hat{\mathcal{J}}_{q}^{\mu}(\mathcal{X}), \hat{\mathcal{J}}_{q^{\prime} \mu}(0)\right]\right\rangle_{T}
$$

is the spectral function corresponding to the vector current. (The metric convention $\mathcal{K} \cdot \mathcal{X}=$ $k_{0} x_{0}-\mathbf{k} \cdot \mathbf{x}$ is assumed.) The spectral function can in turn be expressed as the cut of the corresponding Euclidean correlator,

$$
\rho_{q q^{\prime}}(\mathcal{K})=\operatorname{Im}\left[\Pi_{\mathrm{E} q q^{\prime}}(K)\right]_{k_{n} \rightarrow-i\left[k_{0}+i 0^{+}\right]} .
$$

Here $K=\left(k_{n}, \mathbf{k}\right)$ denotes a Matsubara four-momentum, with $K^{2}=k_{n}^{2}+k^{2}, k \equiv|\mathbf{k}|$.

The Euclidean correlator can be computed with standard path integral techniques. After carrying out the Dirac algebra and making use of substitutions of integration variables, it can be expressed as a sum of a finite number of "master" structures. Up to 2-loop level, the expression reads

$$
\begin{aligned}
\Pi_{\mathrm{E} q q^{\prime}}(K)= & 2(D-2) N_{\mathrm{c}} \delta_{q q^{\prime}} \sum_{\{P\}}\left[\frac{K^{2}}{P^{2}(P-K)^{2}}-\frac{2}{P^{2}}\right] \\
+ & 4(D-2) g^{2} N_{\mathrm{c}} C_{\mathrm{F}} \delta_{q q^{\prime}}\left\{\sum_{\{P\} Q}\left[\frac{D-2}{Q^{2} P^{4}}-\frac{2}{Q^{2} P^{2}(P-K)^{2}}-\frac{(D-2) K^{2}}{Q^{2} P^{4}(P-K)^{2}}\right]\right. \\
& +\sum_{\{P Q\}}\left[-\frac{D-2}{Q^{2} P^{4}}+\frac{D-4}{Q^{2} P^{2}(Q-P)^{2}}+\frac{2}{Q^{2} P^{2}(P-K)^{2}}+\frac{(D-2) K^{2}}{Q^{2} P^{4}(P-K)^{2}}\right. \\
& -\frac{D-4}{Q^{2}(Q-P)^{2}(P-K)^{2}}+\frac{\frac{1}{2}(D-7) K^{2}}{P^{2}(P-K)^{2} Q^{2}(Q-K)^{2}} \\
& \left.\left.+\frac{2(D-2) K \cdot Q-(D-6) K^{2}}{Q^{2} P^{2}(Q-P)^{2}(P-K)^{2}}-\frac{K^{4}}{Q^{2} P^{2}(Q-P)^{2}(Q-K)^{2}(P-K)^{2}}\right]\right\} .
\end{aligned}
$$

Here $D$ is the dimensionality of space-time, and $C_{\mathrm{F}} \equiv\left(N_{\mathrm{c}}^{2}-1\right) /\left(2 N_{\mathrm{c}}\right)$. According to standard conventions, $\mathbb{E}_{\{P\}}$ denotes a sum-integral with fermionic Matsubara momenta, and $\mathscr{E}_{P}$ one with bosonic Matsubara momenta. (The generalization of eq. (2.4) to a finite quark mass can be found in ref. [33].)

The different terms of eq. (2.4) can be referred to as master sum-integrals; their cuts, defined in accordance with eq. (2.3), are the corresponding master spectral functions. In 


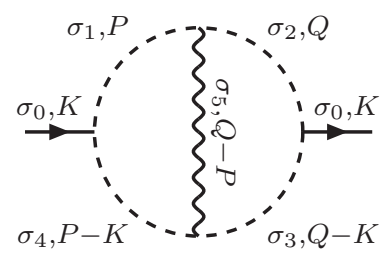

Figure 2. The master Feynman diagram considered, defined by eq. (2.5). The integration measure corresponds to a Matsubara sum-integral, and the spectral function is the discontinuity (imaginary part) of the result once the external Matsubara frequency is analytically continued as $k_{n} \rightarrow-i\left[k_{0}+\right.$ $i 0^{+}$. Dashed lines represent massless propagators; the wiggly line is regulated through a small mass parameter, $\lambda$, for intermediate stages of the computation. The internal and external lines could be either fermions or bosons [denoted by $\sigma_{\mu}=-1$ and +1 , respectively, with $\mu \in\{0, \ldots, 5\}$ ], however fermion number conservation is assumed at each vertex.

particular, the most complicated master sum-integral (the only one with 5 different propagators) is defined from now on as

$$
\mathcal{I}_{\mathbf{j}}(K) \equiv \lim _{\lambda \rightarrow 0} \mathcal{f}_{P Q} \frac{K^{4}}{Q^{2} P^{2}\left[(Q-P)^{2}+\lambda^{2}\right](Q-K)^{2}(P-K)^{2}},
$$

and is illustrated graphically in figure 2. Note that a mass regulator $\lambda$ has been introduced for intermediate stages of the computation. The subscript $(\ldots)_{\mathrm{j}}$ corresponds to the labelling of the various masters in ref. [16]. The statistics of $P, Q$ are meant to be interpreted openly, and are labelled by the indices $\sigma_{0} \ldots \sigma_{5}$ as shown in figure 2 .

The indices $\sigma_{0} \ldots \sigma_{5}$ take the value +1 for bosons and -1 for fermions. Assuming fermion number conservation at each vertex, only three of the indices are independent; we can choose $\sigma_{1}, \sigma_{4}, \sigma_{5}$ to play this role. Then

$$
\sigma_{0}=\sigma_{1} \sigma_{4} ; \quad \sigma_{2}=\sigma_{1} \sigma_{5} ; \quad \sigma_{3}=\sigma_{4} \sigma_{5} .
$$

The case appearing in eq. (2.4) corresponds to $\left(\sigma_{1} \sigma_{4} \sigma_{5}\right)=(--+)$, and a numerical evaluation for the corresponding spectral function, defined in eq. (3.1), is shown in figure 8 below [along the curve in the $\left(k, k_{0}\right)$-plane shown in figure 1].

A completely different physics application, leading to the same master spectral functions but with (partly) different statistics, is that of right-handed neutrino production in the Early Universe. Again, a gauge-invariant Euclidean correlator can be defined,

$$
\Pi_{\mathrm{E}}(K) \equiv \operatorname{Tr}\left\{i K K\left[\int_{0}^{1 / T} \mathrm{~d} \tau \int_{\mathbf{x}} e^{i K \cdot X}\left\langle\left(\tilde{\phi}^{\dagger} a_{\mathrm{L}} \ell\right)(X)\left(\bar{\ell} a_{\mathrm{R}} \tilde{\phi}\right)(0)\right\rangle_{T}\right]\right\}
$$

where $X \equiv(\tau, \mathbf{x}) ; \ell$ is a lepton doublet; $\tilde{\phi}$ is a Higgs doublet; and $a_{\mathrm{L}}, a_{\mathrm{R}}$ are chiral projectors. The production rate now reads

$$
\frac{\mathrm{d} N_{\nu_{\mathrm{R}}}(\mathcal{K})}{\mathrm{d}^{4} \mathcal{X} \mathrm{d}^{3} \mathbf{k}}=\frac{2\left|h_{\nu}\right|^{2}}{(2 \pi)^{3} k_{0}} n_{\mathrm{F}}\left(k_{0}\right) \rho(\mathcal{K})
$$


where $n_{\mathrm{F}}$ is the Fermi distribution; $h_{\nu}$ a bare neutrino Yukawa coupling; and $k_{0}=$ $\sqrt{k^{2}+M^{2}}$, with $M$ denoting the right-handed neutrino mass. The spectral function $\rho$ is obtained from the cut of the Euclidean correlator $\Pi_{\mathrm{E}}$ just like in eq. (2.3).

The 2-loop expression for $\Pi_{\mathrm{E}}$ can be written in a form analogous to eq. (2.4) [16]:

$$
\begin{aligned}
& \Pi_{\mathrm{E}}(K)=-\sum_{P}\left[\frac{2}{P^{2}}+\frac{2 K^{2}}{P^{2}(P-K)^{2}}\right]+\oiint_{\{P\}} \frac{2}{P^{2}} \\
& +12 \lambda_{h} \oint_{P Q}\left[\frac{1}{Q^{2} P^{4}}-\frac{1}{Q^{2} P^{2}(P-K)^{2}}+\frac{K^{2}}{Q^{2} P^{4}(P-K)^{2}}\right] \\
& +2 N_{\mathrm{c}}\left|h_{t}\right|^{2} \oiint_{P\{Q\}}\left[-\frac{2}{Q^{2} P^{4}}+\frac{1}{Q^{2} P^{2}(Q-P)^{2}}+\frac{2}{Q^{2} P^{2}(P-K)^{2}}-\frac{2 K^{2}}{Q^{2} P^{4}(P-K)^{2}}\right. \\
& \left.-\frac{1}{Q^{2}(Q-P)^{2}(P-K)^{2}}+\frac{K^{2}}{Q^{2} P^{2}(Q-P)^{2}(P-K)^{2}}\right] \\
& +\frac{g_{1}^{2}+3 g_{2}^{2}}{2}\left\{\oiint _ { P Q } \left[\frac{D-1}{Q^{2} P^{4}}-\frac{2}{Q^{2} P^{2}(Q-P)^{2}}-\frac{1}{Q^{2} P^{2}(P-K)^{2}}+\frac{(D-1) K^{2}}{Q^{2} P^{4}(P-K)^{2}}\right.\right. \\
& +\frac{2 K^{2}}{P^{2}(P-K)^{2} Q^{2}(Q-K)^{2}}-\frac{4 K^{2}}{Q^{2} P^{2}(Q-P)^{2}(P-K)^{2}} \\
& \left.+\frac{2 K^{4}}{Q^{2} P^{2}(Q-P)^{2}(Q-K)^{2}(P-K)^{2}}\right] \\
& +\oint_{\{P\} Q}\left[-\frac{D-2}{Q^{2} P^{4}}+\frac{(D-2) K^{2}}{Q^{2} P^{4}(P-K)^{2}}+\frac{2(D-2) K \cdot Q-4 K^{2}}{Q^{2} P^{2}(Q-P)^{2}(P-K)^{2}}\right] \\
& +\oint_{P\{Q\}}\left[\frac{2}{Q^{2} P^{2}(Q-P)^{2}}-\frac{D-2}{Q^{2} P^{2}(P-K)^{2}}\right] \\
& \left.+\sum_{\{P Q\}}\left[\frac{D-2}{Q^{2} P^{4}}-\frac{(D-2) K^{2}}{Q^{2} P^{4}(P-K)^{2}}\right]\right\} \text {. }
\end{aligned}
$$

Here $\lambda_{h}$ is the Higgs self-coupling, $h_{t}$ is the top Yukawa coupling, and $g_{1}, g_{2}$ are the hypercharge and weak gauge coupling, respectively.

The master sum-integrals appearing in eq. (2.9) are identical to those in eq. (2.4), apart from their statistics. Noting that $K$ is now fermionic, the most complicated case, eq. (2.5), appears with the indices $\left(\sigma_{1} \sigma_{4} \sigma_{5}\right)=(+-+)$. A numerical evaluation is presented in figure 8 along the curve in the $\left(k, k_{0}\right)$-plane shown in figure 1.

The two examples discussed should serve as illustrations of concrete applications of the spectral function related to eq. (2.5), but do not exhaust the cases considered in the literature. In particular, physical observables leading to the case $\left(\sigma_{1} \sigma_{4} \sigma_{5}\right)=(+++)$ have been analyzed in some detail for $k=0$ in the relativistic regime in refs. [20,21], and for $k \neq 0$ in the non-relativistic regime in refs. [34-36].

As a final remark we mention that above the physical observable was a Lorentz scalar, and subsequently the master structures are scalars as well. In the right-handed neutrino case it may be of interest to ultimately compute the whole self-energy matrix. In this case 
tensor sum-integrals appear apart from scalar ones; the corresponding results have been worked out in the non-relativistic regime in ref. [37]. A general discussion concerning the thermal tensor basis can be found in ref. [38].

\section{Splitup into real and virtual corrections}

Given eq. (2.5), the corresponding spectral function can be defined as

$$
\rho_{\mathcal{I}_{\mathrm{j}}} \equiv \operatorname{Im}\left\{\mathcal{I}_{\mathrm{j}}\right\}_{k_{n} \rightarrow-i\left[k_{0}+i 0^{+}\right]} .
$$

It is well established at 1-loop level that the cut yields a structure reminiscent of a Boltzmann equation, with scattering amplitudes squared multiplied by appropriate phase space distributions [39]. A similar result applies at 2-loop level, but is somewhat complicated by the fact that now virtual particles also appear. In any case, making use of standard techniques, explained in some detail for instance in appendix A of ref. [40], and shifting four-momenta, the following result can be obtained:

$$
\begin{aligned}
& \frac{2 \rho_{\mathcal{I}_{\mathbf{j}}}(\mathcal{K})}{\mathcal{K}^{4}}=\int_{\mathbf{p}, \mathbf{q}, \mathbf{r}} \frac{(2 \pi)^{4} \delta^{(4)}(-\mathcal{K}+\mathcal{P}+\mathcal{Q}+\mathcal{R})}{8 \epsilon_{p} \epsilon_{q} E_{r}} \\
& \times \mathbb{P}\left\{\frac{\left[1+n_{\sigma_{4}}\left(\epsilon_{p}\right)\right]\left[1+n_{\sigma_{2}}\left(\epsilon_{q}\right)\right]\left[1+n_{\sigma_{5}}\left(E_{r}\right)\right]-n_{\sigma_{4}}\left(\epsilon_{p}\right) n_{\sigma_{2}}\left(\epsilon_{q}\right) n_{\sigma_{5}}\left(E_{r}\right)}{(\mathcal{K}-\mathcal{P})^{2}(\mathcal{K}-\mathcal{Q})^{2}}\right\} \\
& +\int_{\mathbf{p}, \mathbf{q}, \mathbf{r}} \frac{(2 \pi)^{4} \delta^{(4)}(-\mathcal{K}-\mathcal{P}+\mathcal{Q}+\mathcal{R})}{8 \epsilon_{p} \epsilon_{q} E_{r}} \\
& \times \mathbb{P}\left\{\frac{n_{\sigma_{4}}\left(\epsilon_{p}\right)\left[1+n_{\sigma_{2}}\left(\epsilon_{q}\right)\right]\left[1+n_{\sigma_{5}}\left(E_{r}\right)\right]-\left[1+n_{\sigma_{4}}\left(\epsilon_{p}\right)\right] n_{\sigma_{2}}\left(\epsilon_{q}\right) n_{\sigma_{5}}\left(E_{r}\right)}{(\mathcal{K}+\mathcal{P})^{2}(\mathcal{K}-\mathcal{Q})^{2}}\right\} \\
& +\int_{\mathbf{p}, \mathbf{q}, \mathbf{r}} \frac{(2 \pi)^{4} \delta^{(4)}(-\mathcal{K}+\mathcal{P}-\mathcal{Q}+\mathcal{R})}{8 \epsilon_{p} \epsilon_{q} E_{r}} \\
& \times \mathbb{P}\left\{\frac{\left[1+n_{\sigma_{4}}\left(\epsilon_{p}\right)\right] n_{\sigma_{2}}\left(\epsilon_{q}\right)\left[1+n_{\sigma_{5}}\left(E_{r}\right)\right]-n_{\sigma_{4}}\left(\epsilon_{p}\right)\left[1+n_{\sigma_{2}}\left(\epsilon_{q}\right)\right] n_{\sigma_{5}}\left(E_{r}\right)}{(\mathcal{K}-\mathcal{P})^{2}(\mathcal{K}+\mathcal{Q})^{2}}\right\} \\
& +\int_{\mathbf{p}, \mathbf{q}, \mathbf{r}} \frac{(2 \pi)^{4} \delta^{(4)}(-\mathcal{K}+\mathcal{P}+\mathcal{Q}-\mathcal{R})}{8 \epsilon_{p} \epsilon_{q} E_{r}} \\
& \times \mathbb{P}\left\{\frac{\left[1+n_{\sigma_{4}}\left(\epsilon_{p}\right)\right]\left[1+n_{\sigma_{2}}\left(\epsilon_{q}\right)\right] n_{\sigma_{5}}\left(E_{r}\right)-n_{\sigma_{4}}\left(\epsilon_{p}\right) n_{\sigma_{2}}\left(\epsilon_{q}\right)\left[1+n_{\sigma_{5}}\left(E_{r}\right)\right]}{(\mathcal{K}-\mathcal{P})^{2}(\mathcal{K}-\mathcal{Q})^{2}}\right\} \\
& +\int_{\mathbf{p}} \frac{2 \pi \delta\left(-k_{0}+\epsilon_{p k}+\epsilon_{p}\right)}{4 \epsilon_{p} \epsilon_{p k}}\left[1+n_{\sigma_{4}}\left(\epsilon_{p k}\right)+n_{\sigma_{1}}\left(\epsilon_{p}\right)\right] \\
& \times \int_{\mathbf{q}} \mathbb{P}\left\{\left.\frac{\frac{1}{2}+n_{\sigma_{2}}\left(\epsilon_{q}\right)}{2 \epsilon_{q}} \frac{1}{\left[(\mathcal{Q}-\mathcal{P})^{2}-\lambda^{2}\right](\mathcal{Q}-\mathcal{K})^{2}}\right|_{q_{0}= \pm \epsilon_{q}}\right. \\
& +\left.\frac{\frac{1}{2}+n_{\sigma_{3}}\left(\epsilon_{q k}\right)}{2 \epsilon_{q k}} \frac{1}{\left[(\mathcal{Q}-\mathcal{P})^{2}-\lambda^{2}\right] \mathcal{Q}^{2}}\right|_{q_{0}=k_{0} \pm \epsilon_{q k}} \\
& \left.+\left.\frac{\frac{1}{2}+n_{\sigma_{5}}\left(E_{q p}\right)}{2 E_{q p}} \frac{1}{\mathcal{Q}^{2}(\mathcal{Q}-\mathcal{K})^{2}}\right|_{q_{0}=p_{0} \pm E_{q p}}\right\}_{p_{0}=\epsilon_{p}, k_{0}=\epsilon_{p}+\epsilon_{p k}}
\end{aligned}
$$




$$
\begin{gathered}
+\int_{\mathbf{p}} \frac{2 \pi \delta\left(-k_{0}-\epsilon_{p k}+\epsilon_{p}\right)}{4 \epsilon_{p} \epsilon_{p k}}\left[n_{\sigma_{4}}\left(\epsilon_{p k}\right)-n_{\sigma_{1}}\left(\epsilon_{p}\right)\right] \\
\times \int_{\mathbf{q}} \mathbb{P}\left\{\left.\frac{\frac{1}{2}+n_{\sigma_{2}}\left(\epsilon_{q}\right)}{2 \epsilon_{q}} \frac{1}{\left[(\mathcal{Q}-\mathcal{P})^{2}-\lambda^{2}\right](\mathcal{Q}-\mathcal{K})^{2}}\right|_{q_{0}= \pm \epsilon_{q}}\right. \\
+\left.\frac{\frac{1}{2}+n_{\sigma_{3}}\left(\epsilon_{q k}\right)}{2 \epsilon_{q k}} \frac{1}{\left[(\mathcal{Q}-\mathcal{P})^{2}-\lambda^{2}\right] \mathcal{Q}^{2}}\right|_{q_{0}=k_{0} \pm \epsilon_{q k}} \\
\left.+\left.\frac{\frac{1}{2}+n_{\sigma_{5}}\left(E_{q p}\right)}{2 E_{q p}} \frac{1}{\mathcal{Q}^{2}(\mathcal{Q}-\mathcal{K})^{2}}\right|_{q_{0}=p_{0} \pm E_{q p}}\right\}_{p_{0}=\epsilon_{p}, k_{0}=\epsilon_{p}-\epsilon_{p k}} \\
+\left(k_{0} \rightarrow-k_{0}\right)+\left(\sigma_{2} \leftrightarrow \sigma_{1}, \sigma_{4} \leftrightarrow \sigma_{3}\right) .
\end{gathered}
$$

Here $\mathbb{P}$ refers to principal value integration which renders changes of integration variables unproblematic; and

$$
\begin{aligned}
& \mathcal{K} \equiv\left(k_{0}, \mathbf{k}\right), \quad \mathcal{P} \equiv\left(\epsilon_{p}, \mathbf{p}\right), \quad \mathcal{Q} \equiv\left(\epsilon_{q}, \mathbf{q}\right), \quad \mathcal{R} \equiv\left(E_{r}, \mathbf{r}\right), \\
& \epsilon_{p} \equiv|\mathbf{p}|, \quad \epsilon_{p k} \equiv|\mathbf{p}-\mathbf{k}|, \quad E_{r} \equiv \sqrt{r^{2}+\lambda^{2}}, \quad E_{q p} \equiv \sqrt{(\mathbf{q}-\mathbf{p})^{2}+\lambda^{2}} .
\end{aligned}
$$

Furthermore the phase space distributions are defined as

$$
\begin{aligned}
n_{\sigma}(\epsilon) & \equiv \frac{\sigma}{e^{\epsilon / T}-\sigma}, & \sigma & = \pm 1 ; \\
n_{+}(\epsilon) & =n_{\mathrm{B}}(\epsilon) \equiv \frac{1}{e^{\epsilon / T}-1}, & n_{-}(\epsilon) & =-n_{\mathrm{F}}(\epsilon) \equiv \frac{-1}{e^{\epsilon / T}+1} .
\end{aligned}
$$

The notation $\left.(\ldots)\right|_{q_{0}= \pm \epsilon_{q}}$ implies that terms with both signs be summed together. The channels labelled by (r1)-(r4) are referred to as real corrections; (v1)-(v3) as virtual corrections. The corresponding physical processes are illustrated in figure 3 .

The last line of eq. (3.2) adds terms with $k_{0} \rightarrow-k_{0}$, which can be shown to render the spectral function antisymmetric in this exchange. In the following we restrict to $k_{0}>0$, and in this case only the terms (r1)-(r4) and (v1)-(v3) contribute. (The unlabelled virtual corrections below (v3) in eq. (3.2) only contribute in the space-like domain.)

\section{Real corrections}

Many numerical evaluations of phase space integrals like (r1)-(r4) can be found in the literature, but we are not aware of a previous representation as a 2-dimensional integral. As is now demonstrated, a fairly explicit expression can be obtained even for $\lambda \neq 0$.

\subsection{Integration variables}

As is common in particle kinematics, a fruitful approach is to represent a complicated phase space as a convolution of simpler ones [41]. Taking case (r1) from eq. (3.2) as an 
$(\mathrm{r} 1)$

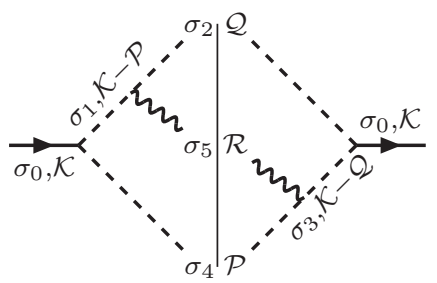

$(\mathrm{r} 2)$

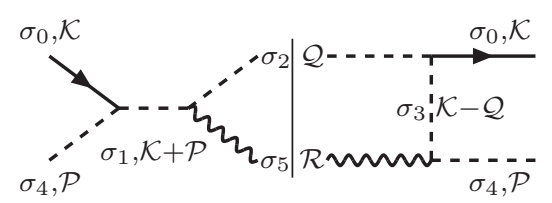

$(\mathrm{r} 3)$

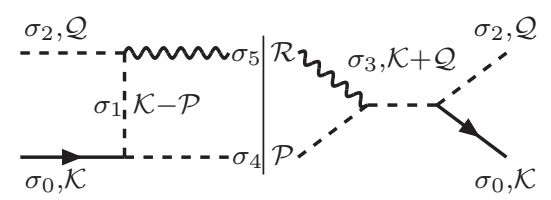

$(\mathrm{r} 4)$

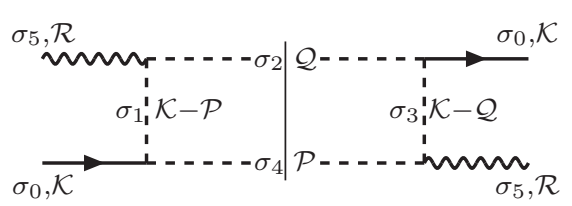

$(\mathrm{v} 1-\mathrm{v} 3)$

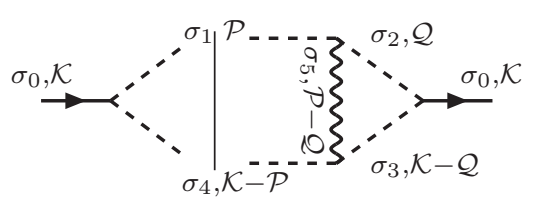

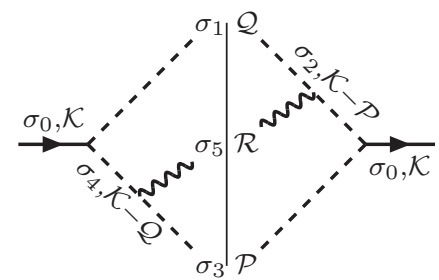
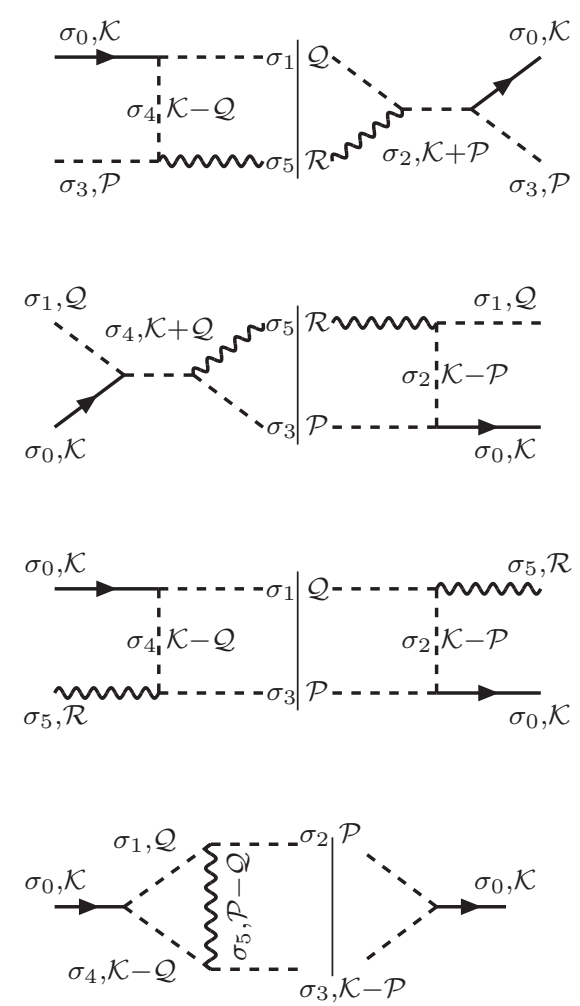

Figure 3. Graphical illustrations of the cut of figure 2, given explicitly in eq. (3.2). Only the decay channels are shown, but as the phase space distributions in eq. (3.2) indicate, the inverse processes are included as well. The processes (r2), (r3), (r4) represent interference terms between $(u, s),(t, s)$ and $(t, u)$ channels. For the virtual corrections the labelling (v1)-(v3) refers to cases in which different internal lines of the closed loop have a thermal weight.

example, we may rewrite it as

$$
\begin{aligned}
\rho_{\mathrm{r} 1} & \equiv \int_{\mathbf{p}, \mathbf{q}, \mathbf{r}} \frac{(2 \pi)^{4} \delta\left(-k_{0}+\epsilon_{p}+\epsilon_{q}+E_{r}\right) \delta^{(3)}(-\mathbf{k}+\mathbf{p}+\mathbf{q}+\mathbf{r})}{8 \epsilon_{p} \epsilon_{q} E_{r}} \Phi_{\mathrm{r} 1}\left(\epsilon_{p}\left|\epsilon_{q}\right| E_{r} \mid \cdot\right) \\
& =\int_{\mathbf{p}, \mathbf{q}} \frac{2 \pi \delta\left(-k_{0}+\epsilon_{p k}+\epsilon_{q}+E_{q p}\right)}{8 \epsilon_{p k} \epsilon_{q} E_{q p}} \Phi_{\mathrm{r} 1}\left(\epsilon_{p k}\left|\epsilon_{q}\right| E_{q p} \mid \cdot\right) \\
& =\int_{-\infty}^{\infty} \mathrm{d} p_{0} \int_{\mathbf{p}, \mathbf{q}} \frac{2 \pi \delta\left(-k_{0}+\epsilon_{p k}+p_{0}\right) \delta\left(-p_{0}+\epsilon_{q}+E_{q p}\right)}{8 \epsilon_{p k} \epsilon_{q} E_{q p}} \Phi_{\mathrm{r} 1}\left(k_{0}-p_{0}\left|\epsilon_{q}\right| p_{0}-\epsilon_{q} \mid \cdot\right),
\end{aligned}
$$


where in the first step we substituted $\mathbf{p} \rightarrow \mathbf{k}-\mathbf{p}$ and integrated over $\mathbf{r}$; and in the second step introduced a variable $p_{0}$ implementing the convolution. The function $\Phi_{\mathrm{r} 1}$ contains phase space distributions as well as propagators; the symbol "." stands for variables not shown explicitly.

The key observation now is that the first three arguments of $\Phi_{\mathrm{r} 1}$, which appear in the phase space distributions, do not contain the variable $p$. Therefore, it is advantageous to take $p_{0}$ and $q$ as the outer integration variables; and $p$ as well as one azimuthal angle that is not fixed by the $\delta$-constraints as the inner ones. The inner integrations contain no phase space distributions and, as we will see, can be carried out explicitly. They also turn out to capture collinear phase space singularities in a manageable form.

The only challenge with this strategy is that it is tedious to work out the limits of the $p$-integration in the plane $\left(p_{0}, q\right)$. Nevertheless, with some work, this challenge is surmountable. Employing the labelling shown in figure 4, the ranges for the case (r1) are $\left[k_{ \pm} \equiv\left(k_{0} \pm k\right) / 2\right]$ :

$$
\begin{aligned}
& \text { (a) }: p \in\left(q-\sqrt{\left(q-p_{0}\right)^{2}-\lambda^{2}}, q+\sqrt{\left(q-p_{0}\right)^{2}-\lambda^{2}}\right) \text {, } \\
& \text { (b) }: p \in\left(q-\sqrt{\left(q-p_{0}\right)^{2}-\lambda^{2}}, 2 k_{+}-p_{0}\right) \text {, } \\
& \text { (ㅁ) }: p \in\left(2 k_{-}-p_{0}, q+\sqrt{\left(q-p_{0}\right)^{2}-\lambda^{2}}\right) \text {, } \\
& \text { (c) : } p \in\left(2 k_{-}-p_{0}, 2 k_{+}-p_{0}\right) \text {, }
\end{aligned}
$$

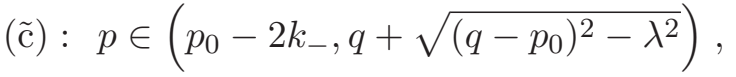

$$
\begin{aligned}
& \text { (d) }: p \in\left(-q+\sqrt{\left(q-p_{0}\right)^{2}-\lambda^{2}}, 2 k_{+}-p_{0}\right) \text {, } \\
& \text { (e) }: p \in\left(p_{0}-2 k_{-}, 2 k_{+}-p_{0}\right) \text {, } \\
& \text { () }): p \in\left(-q+\sqrt{\left(q-p_{0}\right)^{2}-\lambda^{2}}, q+\sqrt{\left(q-p_{0}\right)^{2}-\lambda^{2}}\right) \text {. }
\end{aligned}
$$

As it turns out, the situations $k_{0}<2 k+\sqrt{k^{2}+\lambda^{2}}$ and $k_{0}>2 k+\sqrt{k^{2}+\lambda^{2}}$ need to be handled separately, and this leads to the two cases (c) and ( $\tilde{c})$.

With the ranges at hand, the integrals over the angles

$$
\cos \chi \equiv \frac{\mathbf{p} \cdot \mathbf{k}}{p k}, \quad \cos \theta \equiv \frac{\mathbf{p} \cdot \mathbf{q}}{p q}
$$

can be carried out in eq. (4.1), thereby removing the two $\delta$-functions. We are left with

$$
\rho_{\mathrm{r} 1}=\frac{1}{(4 \pi)^{3} k} \int_{\Omega_{\mathrm{r} 1}} \mathrm{~d} p_{0} \mathrm{~d} q \int_{p_{\min }\left(p_{0}, q\right)}^{p_{\max }\left(p_{0}, q\right)} \mathrm{d} p\left\langle\Phi_{\mathrm{r} 1}\left(k_{0}-p_{0}|q| p_{0}-q \mid \cdot\right)\right\rangle
$$

Here $\langle\ldots\rangle$ refers to an azimuthal average, and $\Omega_{\mathrm{r} 1}$ is composed of the domains (a)-(e) of figure 4.

It is a nice crosscheck to set $\Phi_{\mathrm{r} 1} \rightarrow 1$ and carry out the remaining integrals. In the absence of phase space distributions, the integral is Lorentz-invariant and can alternatively 

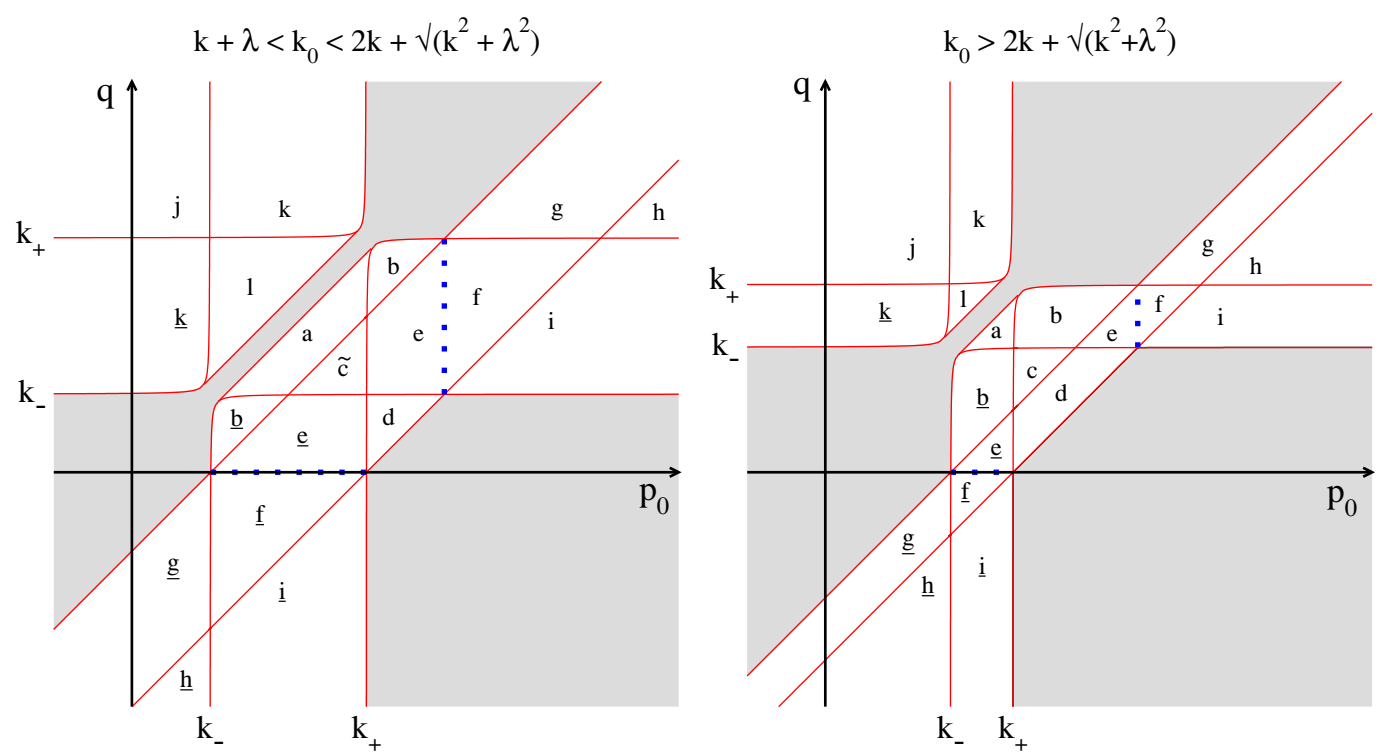

Figure 4. Integration ranges in the $\left(p_{0}, q\right)$-plane for real corrections (for $\left.\lambda=k_{0} / 20\right)$. The delimiting curves are $q=k_{+}-\lambda^{2} /\left[4\left(p_{0}-k_{+}\right)\right], q=k_{-}-\lambda^{2} /\left[4\left(p_{0}-k_{-}\right)\right], q=p_{0}-k_{-}-\lambda^{2} /\left(4 k_{-}\right), q=$ $p_{0}-k_{+}-\lambda^{2} /\left(4 k_{+}\right)$, as well as $q=p_{0} \pm \lambda$. The various channels, separated by the dotted lines and the gap, are in the middle $(\mathrm{r} 1)$; at $p_{0}>k_{0}(\mathrm{r} 2)$; at $q<0(\mathrm{r} 3)$; and at $q>p_{0}(\mathrm{r} 4)$.

be performed in a suitable frame to find a simple result:

$$
\left.\rho_{\mathrm{r} 1}\right|_{\Phi_{\mathrm{r} 1} \rightarrow 1}=\int_{\mathbf{p}, \mathbf{q}, \mathbf{r}} \frac{(2 \pi)^{4} \delta^{(4)} \delta(-\mathcal{K}+\mathcal{P}+\mathcal{Q}+\mathcal{R})}{8 \epsilon_{p} \epsilon_{q} E_{r}}=\frac{1}{(4 \pi)^{3}}\left[\frac{\mathcal{K}^{2}}{4}-\frac{\lambda^{4}}{4 \mathcal{K}^{2}}+\frac{\lambda^{2}}{2} \ln \frac{\lambda^{2}}{\mathcal{K}^{2}}\right] .
$$

This can be reproduced from eq. (4.11), both for $k_{0}<2 k+\sqrt{k^{2}+\lambda^{2}}$ and $k_{0}>2 k+$ $\sqrt{k^{2}+\lambda^{2}}$. However, in the thermal case Lorentz symmetry is not particularly helpful because the plasma defines a special frame, and we need to make use of eqs. (4.2)-(4.9).

The other channels can be handled similarly. For the case (r2), the shift $\mathbf{p} \rightarrow \mathbf{p}-\mathbf{k}$ and a subsequent integration over $\mathbf{r}$ yields

$$
\begin{aligned}
\rho_{\mathrm{r} 2} & \equiv \int_{\mathbf{p}, \mathbf{q}, \mathbf{r}} \frac{(2 \pi)^{4} \delta\left(-k_{0}-\epsilon_{p}+\epsilon_{q}+E_{r}\right) \delta^{(3)}(-\mathbf{k}-\mathbf{p}+\mathbf{q}+\mathbf{r})}{8 \epsilon_{p} \epsilon_{q} E_{r}} \Phi_{\mathrm{r} 2}\left(\epsilon_{p}\left|\epsilon_{q}\right| E_{r} \mid \cdot\right) \\
& =\int_{-\infty}^{\infty} \mathrm{d} p_{0} \int_{\mathbf{p}, \mathbf{q}} \frac{2 \pi \delta\left(-k_{0}-\epsilon_{p k}+p_{0}\right) \delta\left(p_{0}-\epsilon_{q}-E_{q p}\right)}{8 \epsilon_{p k} \epsilon_{q} E_{q p}} \Phi_{\mathrm{r} 2}\left(p_{0}-k_{0}\left|\epsilon_{q}\right| p_{0}-\epsilon_{q} \mid \cdot\right) \\
& =\frac{1}{(4 \pi)^{3} k} \int_{\Omega_{\mathrm{r} 2}} \mathrm{~d} p_{0} \mathrm{~d} q \int_{p_{\min }\left(p_{0}, q\right)}^{p_{\max }\left(p_{0}, q\right)} \mathrm{d} p\left\langle\Phi_{\mathrm{r} 2}\left(p_{0}-k_{0}|q| p_{0}-q \mid \cdot\right)\right\rangle .
\end{aligned}
$$

The domain is displayed in figure 4 , with the ranges

$$
\begin{aligned}
& \text { (f) }: p \in\left(2 k_{+}-p_{0}, p_{0}-2 k_{-}\right), \\
& \text {(g) }: p \in\left(q-\sqrt{\left(q-p_{0}\right)^{2}-\lambda^{2}}, p_{0}-2 k_{-}\right), \\
& \text {(h) }: p \in\left(p_{0}-2 k_{+}, p_{0}-2 k_{-}\right) \\
& \text {(i) }: p \in\left(-q+\sqrt{\left(q-p_{0}\right)^{2}-\lambda^{2}}, p_{0}-2 k_{-}\right) .
\end{aligned}
$$


For the case (r3), the shifts $\mathbf{p} \rightarrow \mathbf{k}-\mathbf{p}, \mathbf{q} \rightarrow-\mathbf{q}$ and a subsequent integration over $\mathbf{r}$ yield

$$
\begin{aligned}
\rho_{\mathrm{r} 3} & \equiv \int_{\mathbf{p}, \mathbf{q}, \mathbf{r}} \frac{(2 \pi)^{4} \delta\left(-k_{0}+\epsilon_{p}-\epsilon_{q}+E_{r}\right) \delta^{(3)}(-\mathbf{k}+\mathbf{p}-\mathbf{q}+\mathbf{r})}{8 \epsilon_{p} \epsilon_{q} E_{r}} \Phi_{\mathrm{r} 3}\left(\epsilon_{p}\left|\epsilon_{q}\right| E_{r} \mid \cdot\right) \\
& =\int_{-\infty}^{\infty} \mathrm{d} p_{0} \int_{\mathbf{p}, \mathbf{q}} \frac{2 \pi \delta\left(-k_{0}+\epsilon_{p k}+p_{0}\right) \delta\left(p_{0}+\epsilon_{q}-E_{q p}\right)}{8 \epsilon_{p k} \epsilon_{q} E_{q p}} \Phi_{\mathrm{r} 3}\left(k_{0}-p_{0}\left|\epsilon_{q}\right| p_{0}+\epsilon_{q} \mid \cdot\right) \\
& =\frac{1}{(4 \pi)^{3} k} \int_{\Omega_{\mathrm{r} 3}} \mathrm{~d} p_{0} \mathrm{~d} q \int_{p_{\min }\left(p_{0}, q\right)}^{p_{\max }\left(p_{0}, q\right)} \mathrm{d} p\left\langle\Phi_{\mathrm{r} 3}\left(k_{0}-p_{0}|-q| p_{0}-q \mid \cdot\right)\right\rangle .
\end{aligned}
$$

In the 2 nd step we substituted formally $\epsilon_{q} \rightarrow-q$, which permits us to represent the domain as displayed in figure 4 , with the ranges

$$
\begin{aligned}
& (\underline{\mathrm{f}}): p \in\left(q+\sqrt{\left(q-p_{0}\right)^{2}-\lambda^{2}},-q+\sqrt{\left(q-p_{0}\right)^{2}-\lambda^{2}}\right), \\
& (\underline{\mathrm{g}}): p \in\left(2 k_{-}-p_{0},-q+\sqrt{\left(q-p_{0}\right)^{2}-\lambda^{2}}\right), \\
& (\underline{\mathrm{h}}): p \in\left(2 k_{-}-p_{0}, 2 k_{+}-p_{0}\right), \\
& (\underline{\mathrm{i}}): p \in\left(q+\sqrt{\left(q-p_{0}\right)^{2}-\lambda^{2}}, 2 k_{+}-p_{0}\right) .
\end{aligned}
$$

Finally, for the case (r4), the shift $\mathbf{p} \rightarrow \mathbf{k}-\mathbf{p}$ and a subsequent integration over $\mathbf{r}$ yields

$$
\begin{aligned}
\rho_{\mathrm{r} 4} & \equiv \int_{\mathbf{p}, \mathbf{q}, \mathbf{r}} \frac{(2 \pi)^{4} \delta\left(-k_{0}+\epsilon_{p}+\epsilon_{q}-E_{r}\right) \delta^{(3)}(-\mathbf{k}+\mathbf{p}+\mathbf{q}-\mathbf{r})}{8 \epsilon_{p} \epsilon_{q} E_{r}} \Phi_{\mathrm{r} 4}\left(\epsilon_{p}\left|\epsilon_{q}\right| E_{r} \mid \cdot\right) \\
& =\int_{-\infty}^{\infty} \mathrm{d} p_{0} \int_{\mathbf{p}, \mathbf{q}} \frac{2 \pi \delta\left(-k_{0}+\epsilon_{p k}+p_{0}\right) \delta\left(p_{0}-\epsilon_{q}+E_{q p}\right)}{8 \epsilon_{p k} \epsilon_{q} E_{q p}} \Phi_{\mathrm{r} 4}\left(k_{0}-p_{0}\left|\epsilon_{q}\right| \epsilon_{q}-p_{0} \mid \cdot\right) \\
& =\frac{1}{(4 \pi)^{3} k} \int_{\Omega_{\mathrm{r} 4}} \mathrm{~d} p_{0} \mathrm{~d} q \int_{p_{\min }\left(p_{0}, q\right)}^{p_{\max }\left(p_{0}, q\right)} \mathrm{d} p\left\langle\Phi_{\mathrm{r} 4}\left(k_{0}-p_{0}|q| q-p_{0} \mid \cdot\right)\right\rangle .
\end{aligned}
$$

The domain is displayed in figure 4 , with the ranges

$$
\begin{aligned}
& \text { (j) }: p \in\left(2 k_{-}-p_{0}, 2 k_{+}-p_{0}\right), \\
& (\mathrm{k}): p \in\left(q-\sqrt{\left(q-p_{0}\right)^{2}-\lambda^{2}}, 2 k_{+}-p_{0}\right), \\
& (\underline{\mathrm{k}}): p \in\left(2 k_{-}-p_{0}, q+\sqrt{\left(q-p_{0}\right)^{2}-\lambda^{2}}\right), \\
& \text { (l) }: p \in\left(q-\sqrt{\left(q-p_{0}\right)^{2}-\lambda^{2}}, q+\sqrt{\left(q-p_{0}\right)^{2}-\lambda^{2}}\right) .
\end{aligned}
$$

\subsection{Crossing symmetry}

At zero temperature, the four channels (r1)-(r4) of figure 3 are related by a crossing symmetry, and it is interesting to see how the presence of phase space distributions in eq. (3.2) changes the situation. Inserting the arguments of $\Phi$ as displayed in 
eqs. (4.11), (4.13), (4.18), (4.23) into expressions obtained from eq. (3.2), we find

$$
\begin{aligned}
\left\langle\Phi_{\mathrm{r} 1}\left(k_{0}-p_{0}|q| p_{0}-q \mid \cdot\right)\right\rangle= & -\left\langle\Phi_{\mathrm{r} 2}\left(p_{0}-k_{0}|q| p_{0}-q \mid \cdot\right)\right\rangle \\
= & -\left\langle\Phi_{\mathrm{r} 3}\left(k_{0}-p_{0}|-q| p_{0}-q \mid \cdot\right)\right\rangle \\
= & -\left\langle\Phi_{\mathrm{r} 4}\left(k_{0}-p_{0}|q| q-p_{0} \mid \cdot\right)\right\rangle \\
= & n_{\sigma_{0}}^{-1}\left(k_{0}\right) n_{\sigma_{4}}\left(k_{0}-p_{0}\right) n_{\sigma_{2}}(q) n_{\sigma_{5}}\left(p_{0}-q\right) \\
& \times \mathbb{P}\left\{\frac{\mathcal{K}^{4}}{2\left(p_{0}^{2}-p^{2}\right)}\left\langle\frac{1}{\left(k_{0}-q\right)^{2}-\epsilon_{q k}^{2}}\right\rangle_{\left(k_{0}-p_{0}|q| p_{0}-q \mid \cdot\right)}\right\},
\end{aligned}
$$

where on the last line the arguments $(\ldots|\ldots| \ldots \mid \cdot)$ refer to $\epsilon_{p k}, \epsilon_{q}$, and $E_{q p}$, respectively, and we made use of the fact that the dependence on $\epsilon_{p k}$ and $E_{q p}$ is quadratic [cf. eqs. (4.35), (4.36), (4.38) below] and that the sign of $q$ inside $\epsilon_{q k}$ plays no role. To arrive at eq. (4.28) the conservation of fermion number [cf. eq. (2.6)], leading to $\sigma_{2} \sigma_{5}=\sigma_{1}$ etc, as well as an identity following from eq. (3.5),

$$
\sigma e^{\epsilon / T} n_{\sigma}(\epsilon)=-n_{\sigma}(-\epsilon)
$$

were assumed. The universal form in eq. (4.28) implies that the azimuthal average $\langle\ldots\rangle$ and the subsequent integration over $p$ only need to be carried out for one single function.

\subsection{Inner integrations}

We now consider the integrations still to be performed, i.e.

$$
I\left(p_{0}, q\right) \equiv \int_{p_{\min }\left(p_{0}, q\right)}^{p_{\max }\left(p_{0}, q\right)} \mathrm{d} p \mathbb{P}\left\{\frac{\mathcal{K}^{4}}{2\left(p_{0}^{2}-p^{2}\right)}\left\langle\frac{1}{\left(k_{0}-q\right)^{2}-\epsilon_{q k}^{2}}\right\rangle_{\left(k_{0}-p_{0}|q| p_{0}-q \mid \cdot\right)}\right\}
$$

Let us start with the azimuthal average. Parametrizing ${ }^{3}$

$$
\begin{aligned}
& \mathbf{p}=p(0,0,1) \\
& \mathbf{k}=k(\sin \chi, 0, \cos \chi) \\
& \mathbf{q}=q(\sin \theta \cos \varphi, \sin \theta \sin \varphi, \cos \theta)
\end{aligned}
$$

the integral over $\varphi$ is readily carried out:

$$
\int_{-\pi}^{\pi} \frac{\mathrm{d} \varphi}{2 \pi} \mathbb{P}\left(\frac{1}{\alpha+\beta \cos \varphi}\right)=\operatorname{Re}\left(\frac{\operatorname{sign}(\alpha)}{\sqrt{\alpha^{2}-\beta^{2}}}\right)
$$

where

$$
\alpha=\left(k_{0}-q\right)^{2}-k^{2}-q^{2}+2 k q \cos \chi \cos \theta, \quad \beta=2 k q \sin \chi \sin \theta .
$$

\footnotetext{
${ }^{3}$ Note that this parametrization can be used both for positive and negative $q$.
} 
Furthermore, according to eq. (4.10), the angles can be written as

$$
\begin{aligned}
\cos \chi=\frac{p^{2}+k^{2}-\epsilon_{p k}^{2}}{2 p k} & =1+\frac{\left(p-p_{0}+2 k_{-}\right)\left(p+p_{0}-2 k_{+}\right)}{2 p k} \\
& =-1+\frac{\left(p-p_{0}+2 k_{+}\right)\left(p+p_{0}-2 k_{-}\right)}{2 p k}, \\
\cos \theta=\frac{p^{2}+q^{2}+\lambda^{2}-E_{q p}^{2}}{2 q p} & =1+\frac{(p-q)^{2}-\left[\left(q-p_{0}\right)^{2}-\lambda^{2}\right]}{2 p q} \\
& =-1+\frac{(p+q)^{2}-\left[\left(q-p_{0}\right)^{2}-\lambda^{2}\right]}{2 p q},
\end{aligned}
$$

where $\epsilon_{p k}, E_{q p}$ were inserted from the arguments shown in eq. (4.30). It can seen that at the boundaries of the $p$-integration, cf. eqs. (4.2)-(4.9), (4.14)-(4.17), (4.19)-(4.22), (4.24)-(4.27), one of the cosines evaluates to \pm 1 , and correspondingly one of the sines vanishes. Therefore, at the boundaries the function $\beta$ given in eq. (4.35) vanishes; this observation will turn out to be useful in a moment.

Inspecting the expressions it is now possible to realize that, in general, the dependence of $\alpha^{2}-\beta^{2}$ appearing in eq. (4.34) on $p$ is of the form

$$
\alpha^{2}-\beta^{2}=a p^{2}+b+\frac{c}{p^{2}} .
$$

Here $a=\left(k_{0}-q\right)^{2}$ and the other coefficients are more complicated. Remarkably, this functional form implies that the integral defined in eq. (4.30) can be carried out: ${ }^{4}$

$$
\begin{aligned}
I\left(p_{0}, q\right)= & \frac{\mathcal{K}^{4}}{4} \int_{p_{\min }}^{p_{\max }} \frac{2 p \mathrm{~d} p}{p_{0}^{2}-p^{2}} \operatorname{Re}\left\{\frac{\operatorname{sign}(\alpha)}{\sqrt{a p^{4}+b p^{2}+c}}\right\} \\
= & \frac{\mathcal{K}^{4} \operatorname{sign}(\alpha)}{4 \sqrt{a p_{0}^{4}+b p_{0}^{2}+c}}\left\{\ln \left|\frac{p_{0}^{2}-p_{\min }^{2}}{p_{0}^{2}-p_{\max }^{2}}\right|\right. \\
& \left.+\ln \left|\frac{\left(\sqrt{a p_{0}^{4}+b p_{0}^{2}+c}+\sqrt{a p_{\max }^{4}+b p_{\max }^{2}+c}\right)^{2}-a\left(p_{0}^{2}-p_{\max }^{2}\right)^{2}}{\left(\sqrt{a p_{0}^{4}+b p_{0}^{2}+c}+\sqrt{a p_{\min }^{4}+b p_{\min }^{2}+c}\right)^{2}-a\left(p_{0}^{2}-p_{\min }^{2}\right)^{2}}\right|\right\} .
\end{aligned}
$$

Furthermore, both square roots can be simplified: the prefactor contains the function

$$
\begin{aligned}
\mathcal{F}\left(p_{0}, q\right) & \equiv \sqrt{a p_{0}^{4}+b p_{0}^{2}+c} \\
& =\sqrt{\left(q-p_{0}\right)^{2} \mathcal{K}^{4}-\lambda^{2}\left[\mathcal{K}^{2}+4 q p_{0}-2 k_{0}\left(q+p_{0}\right)\right] \mathcal{K}^{2}+\lambda^{4} k^{2}}
\end{aligned}
$$

whereas, as already mentioned in connection with eqs. (4.36)-(4.39), the function $\beta$ vanishes for $p=p_{\min }$ and $p=p_{\max }$. Therefore, according to eq. (4.40),

$$
\sqrt{a p_{\max }^{4}+b p_{\max }^{2}+c}=p_{\max }\left|\alpha\left(p_{\max }\right)\right|
$$

and correspondingly for $p_{\min }$, where $\alpha$ is the function from eq. (4.35).

\footnotetext{
${ }^{4}$ The function $\alpha$ can be positive or negative but not change its sign within the ranges considered.
} 
To summarize, all spectral functions corresponding to real corrections have 2dimensional integral representations:

$$
\begin{aligned}
\rho_{\mathrm{r} 1}= & \frac{\pi \mathcal{K}^{4} n_{\sigma_{0}}^{-1}\left(k_{0}\right)}{(4 \pi)^{4} k} \int_{\Omega_{\mathrm{r} 1}} \mathrm{~d} p_{0} \mathrm{~d} q n_{\sigma_{4}}\left(k_{0}-p_{0}\right) n_{\sigma_{2}}(q) n_{\sigma_{5}}\left(p_{0}-q\right) \\
& \times \frac{\operatorname{sign}(\alpha)}{\mathcal{F}\left(p_{0}, q\right)}\left\{\ln \left|\frac{p_{0}^{2}-p_{\min }^{2}}{p_{0}^{2}-p_{\max }^{2}}\right|+\ln \left|\frac{\left[\mathcal{F}\left(p_{0}, q\right)+\left|p_{\max } \alpha\right|\right]^{2}-\left(q-k_{0}\right)^{2}\left(p_{0}^{2}-p_{\max }^{2}\right)^{2}}{\left[\mathcal{F}\left(p_{0}, q\right)+\left|p_{\min } \alpha\right|\right]^{2}-\left(q-k_{0}\right)^{2}\left(p_{0}^{2}-p_{\min }^{2}\right)^{2}}\right|\right\},
\end{aligned}
$$

and correspondingly for $\rho_{\mathrm{r} 2}, \rho_{\mathrm{r} 3}$ and $\rho_{\mathrm{r} 4}$. It can be recalled from eq. (4.28) that the other channels come with an overall minus sign, and we also find that $\operatorname{sign}(\alpha)=-1$ for the channels (r2) and (r4). The integration range $\Omega_{\mathrm{r} 1}+\Omega_{\mathrm{r} 2}+\Omega_{\mathrm{r} 3}+\Omega_{\mathrm{r} 4}$ is as given in figure 4 .

Although well suited for numerical handling, the integral representation in eq. (4.44) remains fairly complicated in practice as long as $\lambda \neq 0$. For $\lambda \rightarrow 0$, the expression simplifies but is also logarithmically divergent. However, when we sum the result together with virtual corrections, to which we now turn, the divergences cancel; the resulting expressions are presented in section 6.

\section{Virtual corrections}

For $k_{0}>k>0$, the virtual corrections are contained within the terms denoted by (v1)(v3) in eq. (3.2), as well as in their reflections $\left(\sigma_{2} \leftrightarrow \sigma_{1}, \sigma_{4} \leftrightarrow \sigma_{3}\right)$. Each of the terms is factorized into two structures. In the first one the angular integration is immediately doable, and thereby we can simplify the radial integration measure into

$$
\int_{\mathbf{p}} \frac{2 \pi \delta\left(-k_{0}+\epsilon_{p}+\epsilon_{p k}\right)}{4 \epsilon_{p} \epsilon_{p k}}\left[1+n_{\sigma_{4}}\left(\epsilon_{p k}\right)+n_{\sigma_{1}}\left(\epsilon_{p}\right)\right]=\frac{n_{\sigma_{0}}^{-1}\left(k_{0}\right)}{8 \pi k} \int_{k_{-}}^{k_{+}} \mathrm{d} p n_{\sigma_{4}}\left(k_{0}-p\right) n_{\sigma_{1}}(p) .
$$

As far as the q-integrals are concerned, their angular parts can be carried out with the help of a Feynman parameter, $s \in(0,1) .{ }^{5}$ Considering first the case (v1), we note that

$$
s\left[(\mathcal{Q}-\mathcal{P})^{2}-\lambda^{2}\right]+(1-s)(\mathcal{Q}-\mathcal{K})^{2}=m_{s}^{2}+2 \mathbf{q} \cdot \mathbf{e}_{s},
$$

where $m_{s}^{2}=(1-s) \mathcal{K}^{2}-s \lambda^{2}-2 q_{0}\left[s p+(1-s) k_{0}\right]$ and $\mathbf{e}_{s} \equiv s \mathbf{p}+(1-s) \mathbf{k}$. Denoting by $z$ the angle between $\mathbf{q}$ and $\mathbf{e}_{s}$, we trivially get

$$
\int_{-1}^{+1} \frac{\mathrm{d} z}{2} \frac{1}{\left(m_{s}^{2}+2 q e_{s} z\right)^{2}}=\frac{1}{m_{s}^{4}-4 q^{2} e_{s}^{2}} .
$$

Furthermore, by making use of the constraint $k_{0}=p+\epsilon_{p k}$ implied by eq. (5.1), the length of $\mathbf{e}_{s}$ is given by $e_{s}^{2}=\left[s p+(1-s) k_{0}\right]^{2}-(1-s) \mathcal{K}^{2}$. The denominator in eq. (5.3) is thus a 2nd order polynomial in $s$, and the integral over $s$ is also doable:

$$
\int_{0}^{1} \frac{\mathrm{d} s}{\mu s^{2}-2 \nu s+\rho}=\frac{1}{2 \sqrt{\nu^{2}-\mu \rho}} \ln \frac{\nu-\rho-\sqrt{\nu^{2}-\mu \rho}}{\nu-\rho+\sqrt{\nu^{2}-\mu \rho}} .
$$

\footnotetext{
${ }^{5}$ The vacuum part, which is represented by the factors $\frac{1}{2}$ in eq. (3.2), could be integrated explicitly by making use of Lorentz invariance (the result is shown in eq. (5.10) below), however for us it is convenient to handle it together with the thermal contributions. For a number of other master spectral functions the vacuum part is divergent at large $|\mathbf{q}|$, but it turns out that even in those cases it is convenient to handle it together with the thermal contributions for moderate $|\mathbf{q}| \lesssim k_{0}$; only the asymptotics at $|\mathbf{q}| \gg k_{0}$ needs to be handled separately with a proper ultraviolet regularization.
} 
As a final step, the terms with $q_{0}= \pm \epsilon_{q}$ can formally be combined by making use of

$$
\frac{1}{2}+n_{\sigma_{2}}(-q)=-\left[\frac{1}{2}+n_{\sigma_{2}}(q)\right] .
$$

Thereby the term (v1) can be cast in the form

$$
\begin{aligned}
\rho_{\mathrm{v} 1}= & \frac{\pi \mathcal{K}^{4} n_{\sigma_{0}}^{-1}\left(k_{0}\right)}{(4 \pi)^{4} k} \int_{k_{-}}^{k_{+}} \mathrm{d} p n_{\sigma_{4}}\left(k_{0}-p\right) n_{\sigma_{1}}(p) \int_{-\infty}^{\infty} \mathrm{d} q\left[\frac{1}{2}+n_{\sigma_{2}}(q)\right] \\
& \times \frac{1}{\mathcal{F}(p, q)} \ln \left|\frac{2 \mathcal{K}^{2} q(q-p)-\lambda^{2}\left(\mathcal{K}^{2}-2 k_{0} q\right)+2 q \mathcal{F}(p, q)}{2 \mathcal{K}^{2} q(q-p)-\lambda^{2}\left(\mathcal{K}^{2}-2 k_{0} q\right)-2 q \mathcal{F}(p, q)}\right|,
\end{aligned}
$$

where $\mathcal{F}$ is the same function as appeared in the real corrections, given by eq. (4.42). The argument of the square root in $\mathcal{F}$ is always positive, so that the integrand is well-defined; ${ }^{6}$ however, the vacuum part of the $q$-integral in eq. (5.6) is divergent on its own, and the integral should only be carried out for the sum of the three terms (v1)-(v3).

The second structure (v2) can be reduced to the first one by substituting variables as $\mathbf{p} \rightarrow \mathbf{k}-\mathbf{p}, \mathbf{q} \rightarrow \mathbf{k}-\mathbf{q}$ in eq. (3.2). Effectively, this corresponds to an interchange $\sigma_{4} \leftrightarrow \sigma_{1}$ in eq. (5.6). However, we can subsequently also substitute $p \rightarrow k_{0}-p, q \rightarrow k_{0}-q$, and given that $\mathcal{F}(p, q)$ of eq. (4.42) is invariant in this transformation, the result reads

$$
\begin{aligned}
\rho_{\mathrm{v} 2}= & \frac{\pi \mathcal{K}^{4} n_{\sigma_{0}}^{-1}\left(k_{0}\right)}{(4 \pi)^{4} k} \int_{k_{-}}^{k_{+}} \mathrm{d} p n_{\sigma_{4}}\left(k_{0}-p\right) n_{\sigma_{1}}(p) \int_{-\infty}^{\infty} \mathrm{d} q\left[\frac{1}{2}+n_{\sigma_{3}}\left(k_{0}-q\right)\right] \\
& \times \frac{1}{\mathcal{F}(p, q)} \ln \left|\frac{2 \mathcal{K}^{2}\left(q-k_{0}\right)(q-p)-\lambda^{2}\left[\mathcal{K}^{2}+2 k_{0}\left(q-k_{0}\right)\right]+2\left(k_{0}-q\right) \mathcal{F}(p, q)}{2 \mathcal{K}^{2}\left(q-k_{0}\right)(q-p)-\lambda^{2}\left[\mathcal{K}^{2}+2 k_{0}\left(q-k_{0}\right)\right]-2\left(k_{0}-q\right) \mathcal{F}(p, q)}\right| .
\end{aligned}
$$

For the third term (v3), we can substitute $\mathbf{q} \rightarrow \mathbf{p}-\mathbf{q}$ in eq. (3.2). Eq. (5.2) gets replaced with

$$
s(\mathcal{P}-\mathcal{Q}-\mathcal{K})^{2}+(1-s)(\mathcal{P}-\mathcal{Q})^{2}=m_{s}^{2}+2 \mathbf{q} \cdot \mathbf{e}_{s},
$$

where now $m_{s}^{2}=\lambda^{2}-2 q_{0}\left(p-s k_{0}\right), \mathbf{e}_{s} \equiv \mathbf{p}-s \mathbf{k}$, and $q_{0}= \pm E_{q}$. The subsequent steps go as before, noting that $e_{s}^{2}=p^{2}+s\left(\mathcal{K}^{2}-2 p k_{0}\right)+s^{2} k^{2}$. Afterwards, it is convenient to again return to the "original" variables; this can be implemented by first taking $E_{q}$ as an integration variable instead of $q$, and then introducing a "new" $q$ as $E_{q}=|p-q|$. In this way the result can be cast in a form reminiscent of eqs. (5.6), (5.7):

$$
\begin{aligned}
\rho_{\mathrm{v} 3}= & \frac{\pi \mathcal{K}^{4} n_{\sigma_{0}}^{-1}\left(k_{0}\right)}{(4 \pi)^{4} k} \int_{k_{-}}^{k_{+}} \mathrm{d} p n_{\sigma_{4}}\left(k_{0}-p\right) n_{\sigma_{1}}(p)\left[\int_{-\infty}^{p-\lambda}+\int_{p+\lambda}^{\infty}\right] \mathrm{d} q\left|\frac{1}{2}+n_{\sigma_{5}}(p-q)\right| \\
& \times \frac{1}{\mathcal{F}(p, q)} \ln \left|\frac{\mathcal{K}^{2}(q-p)^{2}-\lambda^{2}\left[\mathcal{K}^{2}+2 q p-k_{0}(q+p)\right]-\frac{\lambda^{4}}{2}-\sqrt{(q-p)^{2}-\lambda^{2}} \mathcal{F}(p, q)}{\mathcal{K}^{2}(q-p)^{2}-\lambda^{2}\left[\mathcal{K}^{2}+2 q p-k_{0}(q+p)\right]-\frac{\lambda^{4}}{2}+\sqrt{(q-p)^{2}-\lambda^{2}} \mathcal{F}(p, q)}\right| .
\end{aligned}
$$

The expressions in eqs. (5.6), (5.7), (5.9) contain no expansion with respect to $\lambda$. We have crosschecked numerically in the small- $\lambda$ regime that picking up the vacuum factors

\footnotetext{
${ }^{6}$ It may be noted, however, that the integrand has a non-trivial structure at small $q$, with the argument of the logarithm having a zero at $q=-\lambda^{2} /(4 p)$. This (integrable) singularity cancels against a corresponding one from real corrections, related to structure near the boundaries between the regimes (r1) and (r3) in figure 4 , cf. footnote 7 .
} 
(the " $\frac{1}{2}$ " $\mathrm{s}$ if the $n$ 's are expressed with positive arguments), their sum is integrable and reproduces within the achieved resolution a known Lorentz-invariant integral:

$$
\begin{array}{rl}
\int_{Q} & \mathbb{P}\left\{\frac{1}{\left[(Q-P)^{2}+\lambda^{2}\right] Q^{2}(Q-K)^{2}}\right\}_{P^{2}=0, K^{2}=-\mathcal{K}^{2}, K \cdot P=-\frac{\mathcal{K}^{2}}{2}} \\
& =\frac{1}{(4 \pi)^{2} \mathcal{K}^{2}}\left[\frac{\pi^{2}}{6}-\frac{1}{2} \ln ^{2}\left(\frac{\lambda^{2}}{\mathcal{K}^{2}+\lambda^{2}}\right)-\operatorname{Li}_{2}\left(\frac{\lambda^{2}}{\mathcal{K}^{2}+\lambda^{2}}\right)\right] .
\end{array}
$$

\section{Cancellation of divergences}

The results in sections 4 and 5 are both divergent if we attempt to send $\lambda \rightarrow 0$ (cf. eq. (5.10) for the vacuum part). Their sum, however, remains finite as we now demonstrate.

The expressions obtained have two kinds of "singularities". In terms of figure 4, with variables suitably renamed for virtual corrections, the integrands have a non-trivial structure around the boundaries separating the different channels, i.e. $q=p_{0}, q=0$, and $p_{0}=k_{0}$. In addition, away from the boundaries, the integrands in general diverge as $\lambda \rightarrow 0$.

The strategy we adopt is to stay away from the boundaries, for instance by setting a band of width $\delta$ around them, and to take the limit $\lambda \rightarrow 0$ within the domains. ${ }^{7}$ We then verify the cancellation of the corresponding divergences within the domains, and that the resulting integrand remains integrable even after ultimately setting $\delta \rightarrow 0$.

Turning first to the real corrections, eq. (4.44), we simplify the notation from now on by renaming

$$
p_{0} \rightarrow p
$$

Then the following expressions are obtained for the integrand of eq. (4.44) inside the domains of figure 4 :

$$
\begin{aligned}
& \frac{\operatorname{sign}(\alpha)}{\mathcal{F}(p, q)}\{\ldots\} \stackrel{\lambda \rightarrow 0}{\rightarrow} \quad(\mathrm{a})=-(\mathrm{l}): \frac{1}{(p-q) \mathcal{K}^{2}} \ln \left|\frac{\mathcal{K}^{4}(p-q)^{4}}{\lambda^{4} p q\left(p-k_{0}\right)\left(q-k_{0}\right)}\right|, \\
& \text { (b) : } \frac{1}{(p-q) \mathcal{K}^{2}} \ln \left|\frac{\mathcal{K}^{2}(p-q)^{2}\left(q-k_{+}\right)}{\lambda^{2} q\left(q-k_{0}\right)\left(p-k_{+}\right)}\right| \text {, } \\
& (\underline{\mathrm{b}}): \frac{1}{(p-q) \mathcal{K}^{2}} \ln \left|\frac{\mathcal{K}^{2}(p-q)^{2}\left(p-k_{-}\right)}{\lambda^{2} p\left(p-k_{0}\right)\left(q-k_{-}\right)}\right| \text {, } \\
& (\mathrm{c})=(\mathrm{h})=(\underline{\mathrm{h}})=(\mathrm{j}): \frac{1}{(p-q) \mathcal{K}^{2}} \ln \left|\frac{\left(p-k_{-}\right)\left(q-k_{+}\right)}{\left(p-k_{+}\right)\left(q-k_{-}\right)}\right| \text {, } \\
& (\tilde{\mathrm{c}}): \frac{1}{(p-q) \mathcal{K}^{2}} \ln \left|\frac{\mathcal{K}^{4}(p-q)^{4}}{\lambda^{4} p\left(q-k_{0}\right)\left(p-k_{-}\right)\left(q-k_{+}\right)}\right|,
\end{aligned}
$$

\footnotetext{
${ }^{7}$ Actually this requires a somewhat more careful justification, given that according to eq. (4.28) there is a phase space distribution at each boundary which would diverge if it were bosonic. It turns out that if the integrand of eq. (4.44) is evaluated within the domains adjacent to the boundaries and we make the substitution in eq. (6.1), then it cancels exactly against an integrand in one of the virtual corrections, namely that containing the same potentially divergent phase space distribution. In other words, the 2nd row of eq. (4.44) evaluated within the domains (a) and (l) of figure 4 cancels against the 2 nd row of eq. (5.9);

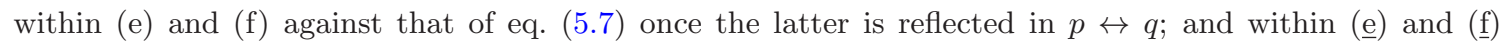
against that of eq. (5.6). Approximate forms of these cancellations can be seen by contrasting eqs. (6.2) and (6.15); (6.8) and (6.14) [with $p \leftrightarrow q$ ]; as well as (6.9) and (6.13).
} 


$$
\begin{array}{r}
(\mathrm{d}): \frac{1}{(p-q) \mathcal{K}^{2}} \ln \left|\frac{q\left(p-k_{0}\right)}{\left(p-k_{+}\right)\left(q-k_{-}\right)}\right|, \\
(\mathrm{e})=-(\mathrm{f}): \frac{1}{(p-q) \mathcal{K}^{2}} \ln \left|\frac{\mathcal{K}^{2}(p-q)^{2}\left(p-k_{0}\right)}{\lambda^{2}\left(q-k_{0}\right)\left(p-k_{-}\right)\left(p-k_{+}\right)}\right|, \\
(\underline{\mathrm{e}})=-(\underline{\mathrm{f}}): \frac{1}{(p-q) \mathcal{K}^{2}} \ln \left|\frac{\mathcal{K}^{2}(p-q)^{2} q}{\lambda^{2} p\left(q-k_{-}\right)\left(q-k_{+}\right)}\right|, \\
-(\mathrm{g})=-(\underline{\mathrm{g}}): \frac{1}{(p-q) \mathcal{K}^{2}} \ln \left|\frac{q\left(p-k_{0}\right)}{\left(p-k_{-}\right)\left(q-k_{+}\right)}\right|, \\
-(\mathrm{i})=-(\underline{\mathrm{k}}): \frac{1}{(p-q) \mathcal{K}^{2}} \ln \left|\frac{\mathcal{K}^{2}(p-q)^{2}\left(q-k_{-}\right)}{\lambda^{2} q\left(q-k_{0}\right)\left(p-k_{-}\right)}\right|, \\
-(\underline{\mathrm{i}})=-(\mathrm{k}): \frac{1}{(p-q) \mathcal{K}^{2}} \ln \left|\frac{\mathcal{K}^{2}(p-q)^{2}\left(p-k_{+}\right)}{\lambda^{2} p\left(p-k_{0}\right)\left(q-k_{+}\right)}\right| .
\end{array}
$$

These are multiplied by phase space distributions as indicated by eq. (4.28), and in addition the symmetrization $\left(\sigma_{2} \leftrightarrow \sigma_{1}, \sigma_{4} \leftrightarrow \sigma_{3}\right)$ from the last line of eq. (3.2) needs to be included.

Similar expressions are obtained for the virtual corrections, eqs. (5.6), (5.7), (5.9):

$$
\begin{aligned}
\frac{1}{\mathcal{F}(p, q)} \ln |\ldots| \stackrel{\lambda \rightarrow 0}{\rightarrow} \quad(\mathrm{v} 1): & \frac{1}{(p-q) \mathcal{K}^{2}} \ln \left|\frac{\lambda^{2} p\left(q-k_{-}\right)\left(q-k_{+}\right)}{\mathcal{K}^{2} q(p-q)^{2}}\right| \\
& (\mathrm{v} 2): \frac{-1}{(p-q) \mathcal{K}^{2}} \ln \left|\frac{\lambda^{2}\left(p-k_{0}\right)\left(q-k_{-}\right)\left(q-k_{+}\right)}{\mathcal{K}^{2}\left(q-k_{0}\right)(p-q)^{2}}\right| \\
& (\mathrm{v} 3): \frac{1}{|p-q| \mathcal{K}^{2}} \ln \left|\frac{\lambda^{4} p q\left(p-k_{0}\right)\left(q-k_{0}\right)}{\mathcal{K}^{4}(p-q)^{4}}\right| .
\end{aligned}
$$

Again the symmetrization $\left(\sigma_{2} \leftrightarrow \sigma_{1}, \sigma_{4} \leftrightarrow \sigma_{3}\right)$ needs to be included.

In order to combine eqs. (6.13)-(6.15) with the real corrections, it is beneficial to rename integration variables. By making use of

$$
n_{\sigma}(-\epsilon)=-1-n_{\sigma}(\epsilon), \quad n_{\sigma}(\epsilon) n_{\tau}(\delta-\epsilon)=n_{\sigma \tau}(\delta)\left[1+n_{\sigma}(\epsilon)+n_{\tau}(\delta-\epsilon)\right]
$$

and recalling the identities $\sigma_{2} \sigma_{5}=\sigma_{1}, \sigma_{3} \sigma_{5}=\sigma_{4}$, following from eq. (2.6), we can write

$$
\begin{aligned}
\frac{1}{2}+n_{\sigma_{2}}(q) & =n_{\sigma_{1}}^{-1}(p) n_{\sigma_{2}}(q) n_{\sigma_{5}}(p-q)-\frac{1}{2}-n_{\sigma_{5}}(p-q), \\
\frac{1}{2}+n_{\sigma_{3}}\left(k_{0}-q\right) & =n_{\sigma_{4}}^{-1}\left(k_{0}-p\right) n_{\sigma_{3}}\left(k_{0}-q\right) n_{\sigma_{5}}(q-p)+\frac{1}{2}+n_{\sigma_{5}}(p-q) .
\end{aligned}
$$

Furthermore, in the first term on the right-hand side of eq. (6.18), in which $n_{\sigma_{3}}\left(k_{0}-q\right)$ appears, we exchange variables as $p \leftrightarrow q$. In the terms from $\left(\sigma_{2} \leftrightarrow \sigma_{1}, \sigma_{4} \leftrightarrow \sigma_{3}\right)$, we can also do this in the terms involving $\frac{1}{2}+n_{\sigma_{5}}(p-q)$ for more symmetry.

The effect of these rewritings is that logarithms of $\lambda$ disappear from terms involving $\frac{1}{2}+n_{\sigma_{5}}(p-q)$ [essentially its coefficient is given by $-(6.13)+(6.14)+(6.15)$ ]. Logarithms of $\lambda$ do not cancel from the coefficients of the first terms of eqs. (6.17), (6.18) but, combining with the other phase space distributions from eqs. (5.6), (5.7), are seen to come with the same "weight functions" as in the real corrections. To be explicit, the virtual corrections $\left[\rho_{\mathrm{v}} \equiv \rho_{\mathrm{v} 1}+\rho_{\mathrm{v} 2}+\rho_{\mathrm{v} 3}+\left(\sigma_{2} \leftrightarrow \sigma_{1}, \sigma_{4} \leftrightarrow \sigma_{3}\right)\right]$ can be represented within the domains shown 


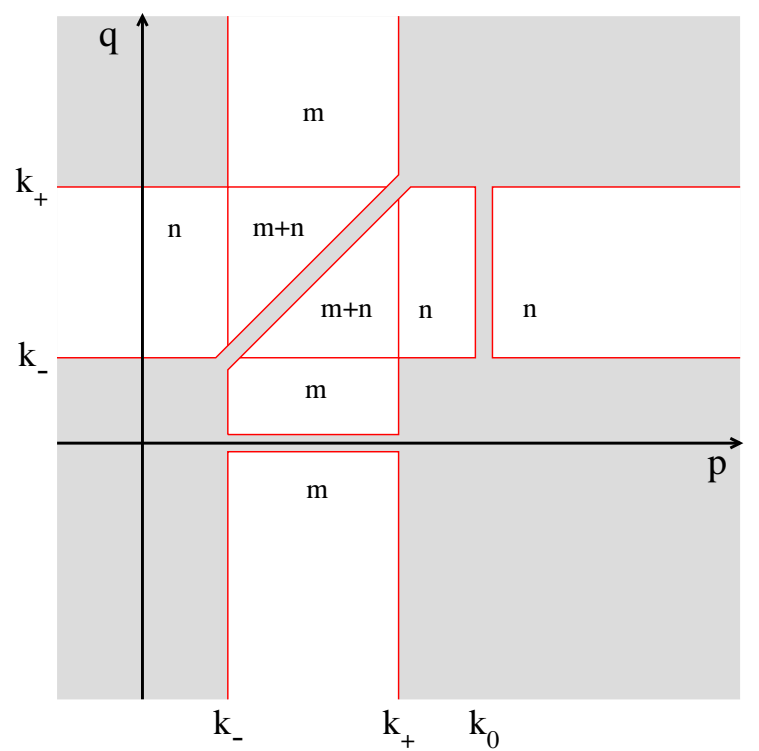

Figure 5. Integration ranges in the $(p, q)$-plane for virtual corrections. The delimiting curves are $p=k_{-}, p=k_{+}, q=k_{-}$, and $q=k_{+}$. Narrow bands have been cut off around the lines $q=p, q=0$ and $p=k_{0}$.

in figure 5 as

$$
\begin{aligned}
\frac{(4 \pi)^{4} k \rho_{\mathrm{v}}(\mathcal{K})}{\pi \mathcal{K}^{4} n_{\sigma_{0}}^{-1}\left(k_{0}\right)}= & \int_{\Omega_{\mathrm{m}}} \mathrm{d} p \mathrm{~d} q\left\{\left[n_{\sigma_{4}}\left(k_{0}-p\right) n_{\sigma_{2}}(q)+n_{\sigma_{3}}\left(k_{0}-p\right) n_{\sigma_{1}}(q)\right] n_{\sigma_{5}}(p-q)\right. \\
& \times \frac{1}{(p-q) \mathcal{K}^{2}} \ln \left|\frac{\lambda^{2} p\left(q-k_{-}\right)\left(q-k_{+}\right)}{\mathcal{K}^{2} q(p-q)^{2}}\right| \\
& +n_{\sigma_{4}}\left(k_{0}-p\right) n_{\sigma_{1}}(p)\left[\frac{1}{2}+n_{\sigma_{5}}(p-q)\right] \\
& \left.\times \frac{2}{(p-q) \mathcal{K}^{2}} \ln \left|\frac{q\left(q-k_{0}\right)}{\left(q-k_{-}\right)\left(q-k_{+}\right)}\right|\right\} \\
+ & \int_{\Omega_{\mathrm{n}}} \mathrm{d} p \mathrm{~d} q\left\{\left[n_{\sigma_{4}}\left(k_{0}-p\right) n_{\sigma_{2}}(q)+n_{\sigma_{3}}\left(k_{0}-p\right) n_{\sigma_{1}}(q)\right] n_{\sigma_{5}}(p-q)\right. \\
& \times \frac{1}{(p-q) \mathcal{K}^{2}} \ln \left|\frac{\lambda^{2}\left(q-k_{0}\right)\left(p-k_{-}\right)\left(p-k_{+}\right)}{\mathcal{K}^{2}\left(p-k_{0}\right)(p-q)^{2}}\right| \\
+ & n_{\sigma_{3}}\left(k_{0}-q\right) n_{\sigma_{2}}(q)\left[\frac{1}{2}+n_{\sigma_{5}}(p-q)\right] \\
& \left.\times \frac{2}{(p-q) \mathcal{K}^{2}} \ln \left|\frac{p\left(p-k_{0}\right)}{\left(p-k_{-}\right)\left(p-k_{+}\right)}\right|\right\}
\end{aligned}
$$

Combining eq. (6.19) with eqs. (6.2)-(6.12), the latter multiplied by phase space distributions according to eq. (4.28), and adding for the real corrections terms from the symmetrization $\left(\sigma_{2} \leftrightarrow \sigma_{1}, \sigma_{4} \leftrightarrow \sigma_{3}\right)$, all logarithms of $\lambda$ are now seen to cancel. The resulting integrand is sufficiently well-behaved around the boundaries to be integrable [cf. eq. (7.9) and section 8]. 

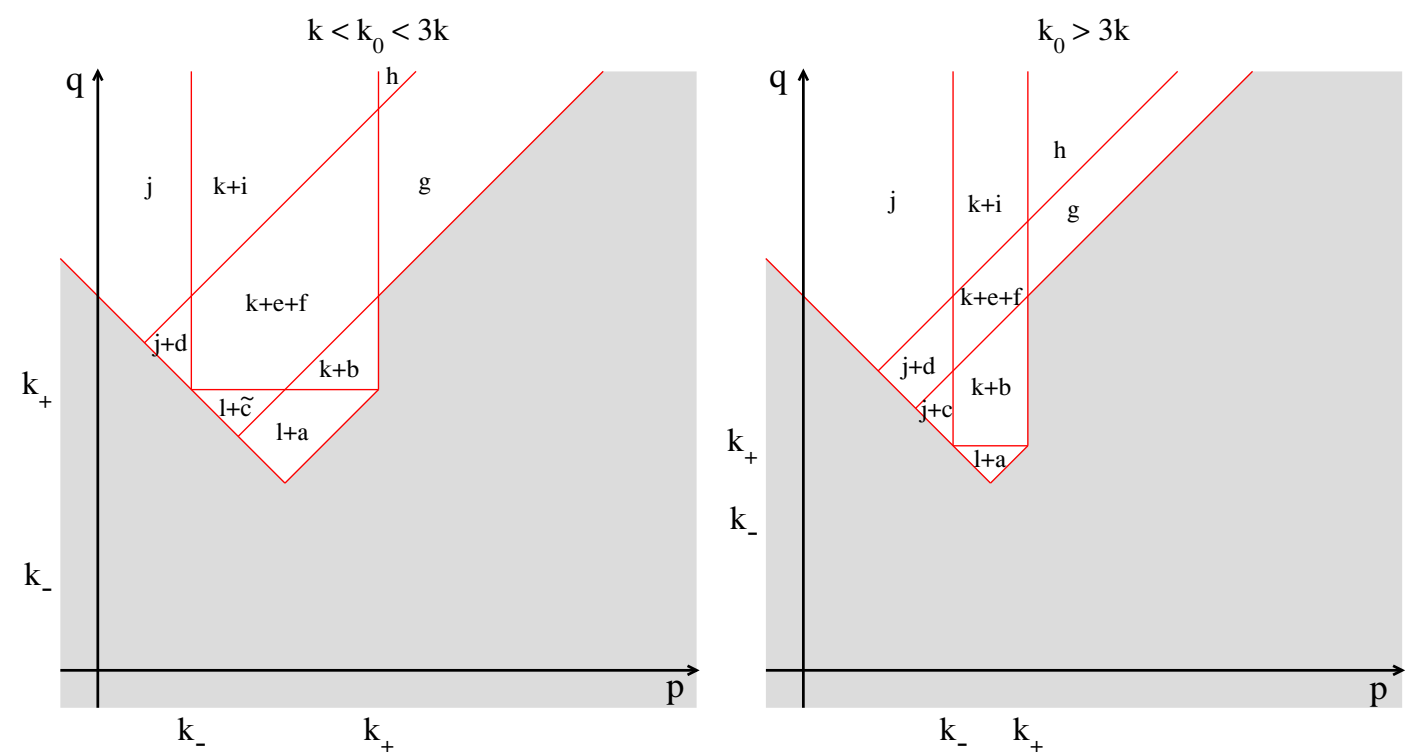

Figure 6. Integration ranges in the $(p, q)$-plane for the final answer, eq. (7.9). The delimiting curves are $p=k_{-}, p=k_{+}, q=p, q=p+k_{-}, q=p+k_{+}, q=k_{0}-p$, as well as $q=k_{+}$.

\section{$7 \quad$ Final result}

In order to collect together a final result, we remove redundant symmetries from the expression. It is suggested already by eq. (4.42) that the two substitutions

$$
p \leftrightarrow k_{0}-q ; \quad p \leftrightarrow q,
$$

corresponding to reflections across the axes $q=k_{0}-p$ and $q=p$, respectively, may be helpful in this respect. Indeed, the first of these symmetries is manifest in the real corrections, and can consequently be seen to transform the "coefficient functions", eqs. (6.2)-(6.12), into each other. The phase space distributions are in general not invariant but now become symmetrized with respect to their indices (concretely, a symmetry in $\left(\sigma_{1} \leftrightarrow \sigma_{4}, \sigma_{2} \leftrightarrow \sigma_{3}\right)$ which was so far hidden becomes explicit).

A symmetry in $p \leftrightarrow q$ is not "inherent" to the expressions. It is useful to make this reflection, however, because it explicitly regulates principal value integrations across $p-q=0$. After these reflections, the integration range is as shown in figure 6 .

To present a final result, we undertake one more rewriting of the phase space distributions. By making use of eqs. (5.5), (6.16), the structures appearing in the real corrections are expressed as

$$
n_{\sigma_{4}}\left(k_{0}-p\right) n_{\sigma_{2}}(q) n_{\sigma_{5}}(p-q)=n_{\sigma_{4}}\left(k_{0}-p\right) n_{\sigma_{1}}(p)\left\{\left[\frac{1}{2}+n_{\sigma_{2}}(q)\right]-\left[\frac{1}{2}+n_{\sigma_{5}}(q-p)\right]\right\}
$$

where $n_{\sigma_{5}}$ has been taken with a positive argument. We then introduce the "weight 
functions"

$$
\begin{aligned}
& \omega_{1} \equiv n_{\sigma_{4}}\left(k_{0}-p\right) n_{\sigma_{1}}(p)\left[\frac{1}{2}+n_{\sigma_{5}}(q-p)\right]+\left(\sigma_{1}, \sigma_{4}, \sigma_{5}\right)+\left(\sigma_{2}, \sigma_{3}, \sigma_{5}\right)+\left(\sigma_{3}, \sigma_{2}, \sigma_{5}\right) \\
& \omega_{2} \equiv n_{\sigma_{4}}\left(k_{0}-q\right) n_{\sigma_{1}}(q)\left[\frac{1}{2}+n_{\sigma_{5}}(q-p)\right]+\left(\sigma_{1}, \sigma_{4}, \sigma_{5}\right)+\left(\sigma_{2}, \sigma_{3}, \sigma_{5}\right)+\left(\sigma_{3}, \sigma_{2}, \sigma_{5}\right) \\
& \omega_{3} \equiv n_{\sigma_{4}}\left(k_{0}-p\right) n_{\sigma_{1}}(p)\left[\frac{1}{2}+n_{\sigma_{2}}(q)\right]+\left(\sigma_{1}, \sigma_{4}, \sigma_{3}\right)+\left(\sigma_{2}, \sigma_{3}, \sigma_{4}\right)+\left(\sigma_{3}, \sigma_{2}, \sigma_{1}\right) \\
& \omega_{4} \equiv n_{\sigma_{4}}\left(k_{0}-q\right) n_{\sigma_{1}}(q)\left[\frac{1}{2}+n_{\sigma_{2}}(p)\right]+\left(\sigma_{1}, \sigma_{4}, \sigma_{3}\right)+\left(\sigma_{2}, \sigma_{3}, \sigma_{4}\right)+\left(\sigma_{3}, \sigma_{2}, \sigma_{1}\right) .
\end{aligned}
$$

To characterize the "coefficient functions" it is convenient to define the ratios

$$
\begin{aligned}
\chi_{-} \equiv \frac{q-k_{-}}{q-k_{0}}, & \chi_{+} \equiv \frac{q-k_{+}}{q-k_{0}}, & \chi_{0} \equiv \frac{q}{q-k_{0}}, \\
\pi_{-} \equiv \frac{p-k_{-}}{p-k_{0}}, & \pi_{+} \equiv \frac{p-k_{+}}{p-k_{0}}, & \pi_{0} \equiv \frac{p}{p-k_{0}} .
\end{aligned}
$$

Then the full result becomes

$$
\begin{aligned}
& \frac{(4 \pi)^{4} k \rho_{\mathcal{I}_{\mathrm{j}}}(\mathcal{K})}{\pi \mathcal{K}^{2} n_{\sigma_{0}}^{-1}\left(k_{0}\right)}= \\
& \quad \int_{\Omega_{1+\mathrm{a}}} \frac{\mathrm{d} p \mathrm{~d} q}{q-p}\left\{\left(\omega_{1}-\omega_{2}\right) \ln \left|\frac{\pi_{-} \pi_{+}}{\chi_{-} \chi_{+}}\right|+\omega_{3} \ln \left|\frac{\chi_{0}^{2}}{\chi_{-} \chi_{+} \pi_{-} \pi_{+}}\right|+\omega_{4} \ln \left|\frac{\chi_{-} \chi_{+} \pi_{-} \pi_{+}}{\pi_{0}^{2}}\right|\right\} \\
& \quad+\int_{\Omega_{\mathrm{k}+\mathrm{b}}} \frac{\mathrm{d} p \mathrm{~d} q}{q-p}\left\{\omega_{1} \ln \left|\frac{\chi_{0} \pi_{+}}{\chi_{-} \chi_{+}^{2}}\right|+\left(\omega_{2}+\omega_{4}\right) \ln \left|\frac{\chi_{-} \pi_{+}}{\pi_{0}}\right|+\omega_{3} \ln \left|\frac{\chi_{0}}{\chi_{-} \pi_{+}}\right|\right\} \\
& \quad+\int_{\Omega_{\mathrm{j}+\mathrm{c}}} \frac{\mathrm{d} p \mathrm{~d} q}{q-p}\left\{\left(\omega_{1}+\omega_{2}-\omega_{3}+\omega_{4}\right) \ln \left|\frac{\chi_{-} \pi_{+}}{\chi_{+} \pi_{-}}\right|\right\} \\
& \quad+\int_{\Omega_{1+\tilde{c}}} \frac{\mathrm{d} p \mathrm{~d} q}{q-p}\left\{\omega_{1} \ln \left|\frac{\pi_{-} \pi_{+}}{\chi_{-} \chi_{+}}\right|+\omega_{2} \ln \left|\frac{\pi_{0} \chi_{+}}{\pi_{-} \pi_{+}^{2}}\right|+\omega_{3} \ln \left|\frac{\chi_{0}^{2}}{\chi_{-} \chi_{+} \pi_{-} \pi_{+}}\right|+\omega_{4} \ln \left|\frac{\chi_{+} \pi_{-}}{\pi_{0}}\right|\right\} \\
& \quad+\int_{\Omega_{\mathrm{j}+\mathrm{d}}} \frac{\mathrm{d} p \mathrm{~d} q}{q-p}\left\{\left(\omega_{1}-\omega_{3}\right) \ln \left|\frac{\chi_{-} \pi_{+}}{\chi_{+} \pi_{-}}\right|+\left(\omega_{2}+\omega_{4}\right) \ln \left|\frac{\pi_{0}}{\chi_{+} \pi_{-}}\right|\right\} \\
& \quad+\int_{\Omega_{\mathrm{k}+\mathrm{e}+\mathrm{f}}} \frac{\mathrm{d} p \mathrm{~d} q}{q-p}\left\{\omega_{1} \ln \left|\frac{\chi_{0} \pi_{+}}{\chi_{-} \chi_{+}^{2}}\right|+\omega_{3} \ln \left|\frac{\chi_{0}}{\chi_{-} \pi_{+}}\right|\right\} \\
& \quad+\int_{\Omega_{\mathrm{g}}} \frac{\mathrm{d} p \mathrm{~d} q}{q-p}\left\{\left(\omega_{2}+\omega_{4}\right) \ln \left|\frac{\pi_{0}}{\chi_{-} \pi_{+}}\right|\right\} \\
& \quad+\int_{\Omega_{\mathrm{h}}} \frac{\mathrm{d} p \mathrm{~d} q}{q-p}\left\{\left(\omega_{2}+\omega_{4}\right) \ln \left|\frac{\chi_{+} \pi_{-}}{\chi_{-} \pi_{+}}\right|\right\} \\
& \quad+\int_{\Omega_{\mathrm{k}+\mathrm{i}}} \frac{\mathrm{d} p \mathrm{~d} q}{q-p}\left\{\omega_{1} \ln \left|\frac{\chi_{0} \pi_{+}}{\chi_{-} \chi_{+}^{2}}\right|+\left(\omega_{2}+\omega_{4}\right) \ln \left|\frac{\chi_{+} \pi_{-}}{\pi_{0}}\right|+\omega_{3} \ln \left|\frac{\chi_{0}}{\chi_{-} \pi_{+}}\right|\right\} \\
& +\int_{\Omega_{\mathrm{j}}} \frac{\mathrm{d} p \mathrm{~d} q}{q-p}\left\{\left(\omega_{1}-\omega_{3}\right) \ln \left|\frac{\chi_{-} \pi_{+}}{\chi_{+} \pi_{-}}\right|\right\}
\end{aligned}
$$

where the $\Omega$ 's denote different domains as labelled in figure 6 . Note that only one among the ranges $\Omega_{\mathrm{j}+\mathrm{c}}$ and $\Omega_{1+\tilde{c}}$ gets realized at a time. 

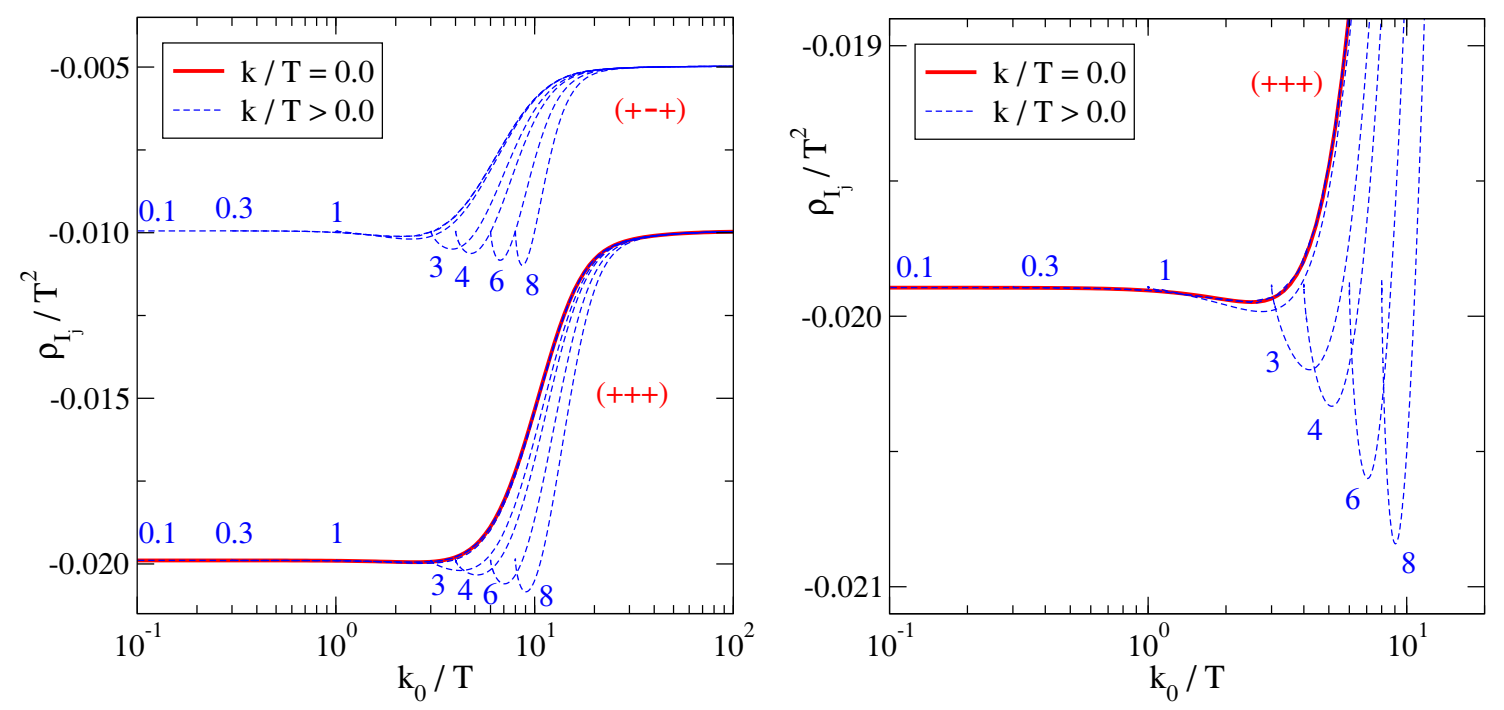

Figure 7. Left: a comparison of the spectral function at non-zero momentum $(k / T=0.1,0.3,1$, $3,4,6,8$ as indicated in the figure) with the zero-momentum limit from eq. (A.57) of ref. [20], for $\left(\sigma_{1} \sigma_{4} \sigma_{5}\right)=(+++)$. For the case $\left(\sigma_{1} \sigma_{4} \sigma_{5}\right)=(+-+)$ only the non-zero momenta are shown. In both cases we have restricted to $k_{0} \geq k+0.001 T$. Right: a magnification of the case $\left(\sigma_{1} \sigma_{4} \sigma_{5}\right)=$ $(+++)$.

\section{Numerical evaluation}

The expression in eq. (7.9) is finite and can be evaluated numerically. In figure 7 the outcome is compared with its limiting value at $k=0$, determined for the case $\left(\sigma_{1} \sigma_{4} \sigma_{5}\right)=$ $(+++)$ in ref. [20]. The results are seen to agree for $k_{0} \gg k$; in fact, even when this inequality is not satisfied, the zero-momentum limit yields a surprisingly good approximation of the full result.

It is important to realize that the spectral function $\rho_{\mathcal{I}_{\mathrm{j}}}$ is in general non-trivial in the vicinity of the light cone, cf. figure 7 , rather than vanishing as $\sim \mathcal{K}^{2}$ as one could expect from dimensional reasons at zero temperature. This fact leads ultimately to the breakdown of the loop expansion for $\mathcal{K}^{2} \lesssim(g T)^{2}$ [2-loop diagrams may give a larger contribution than 1-loop ones despite the overall suppression by $g^{2}$ ], and leads to the need to resum a set of diagrams for obtaining the correct result in the ultrarelativistic regime.

Another comparison can be made with the non-relativistic asymptotics, determined in ref. [16]. The leading term, proportional to $\mathcal{K}^{2}$, corresponds to the zero-temperature limit and vanishes for the spectral function in question. The two first non-zero terms read

$$
\rho_{\mathcal{I}_{\mathrm{j}}}=-\frac{1}{16 \pi} \int_{p}\left\{\frac{\sum_{i=1}^{4} n_{\sigma_{i}}+2 n_{\sigma_{5}}}{p}+p\left[\frac{22}{3} \sum_{i=1}^{4} n_{\sigma_{i}}+4 n_{\sigma_{5}}\right] \frac{k_{0}^{2}+k^{2} / 3}{\mathcal{K}^{4}}\right\}+\mathcal{O}\left(\frac{T^{6}}{\mathcal{K}^{4}}\right)
$$

where

$$
\int_{p} \frac{n_{\mathrm{B}}}{p}=\frac{T^{2}}{12}, \quad \int_{p} \frac{n_{\mathrm{F}}}{p}=\frac{T^{2}}{24}, \quad \int_{p} p n_{\mathrm{B}}=\frac{\pi^{2} T^{4}}{30}, \quad \int_{p} p n_{\mathrm{F}}=\frac{7 \pi^{2} T^{4}}{240} .
$$



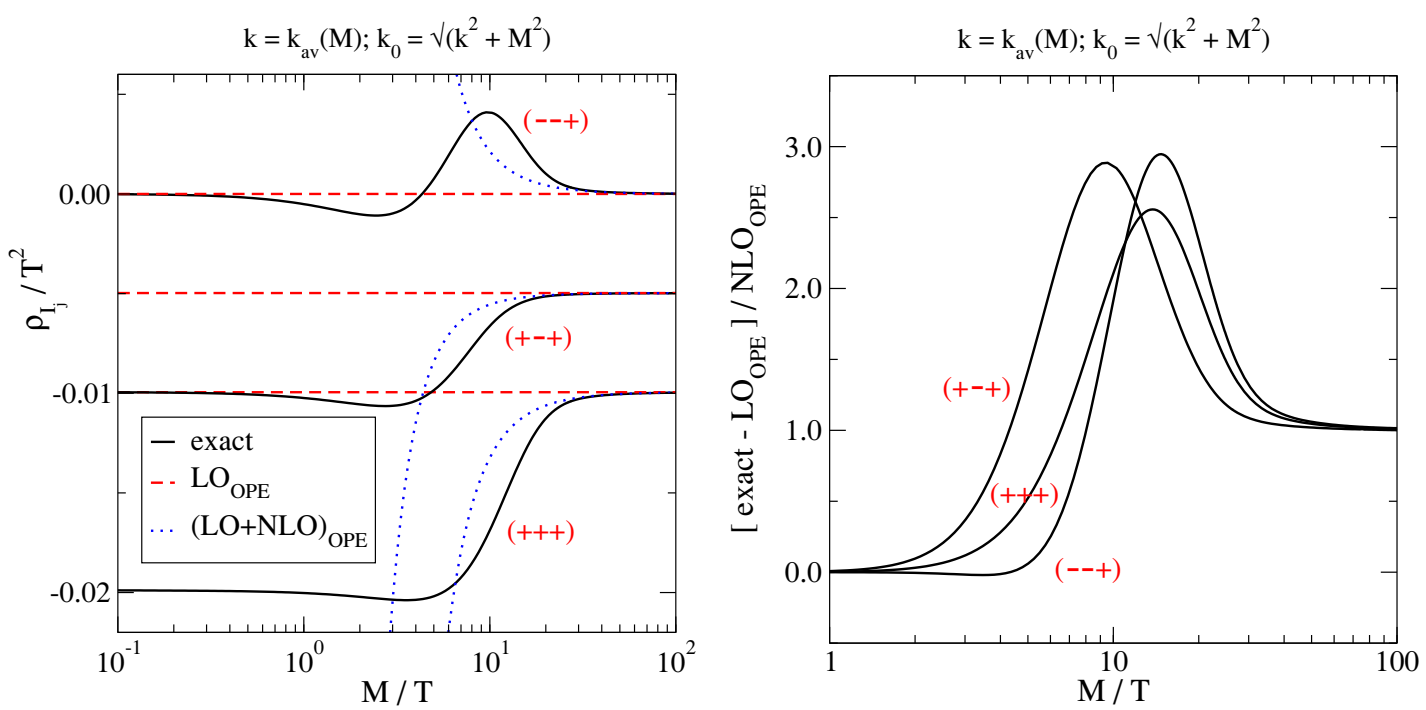

Figure 8. Left: spectral functions for various statistics $\left(\sigma_{1} \sigma_{4} \sigma_{5}\right)$, as a function of $M^{2} \equiv \mathcal{K}^{2}$, with $k \equiv k_{\mathrm{av}}$ determined from eq. (8.3). The results are compared with two orders of OPE asymptotics from eq. (8.1). Right: the ratio [exact $\left.-\mathrm{LO}_{\mathrm{OPE}}\right] / \mathrm{NLO}_{\mathrm{OPE}}$, showing that numerical results agree with the asymptotic ones from eq. (8.1) for $M \gtrsim 30 T$.

In figure 8 the two orders shown in eq. (8.1) are referred to as " $\mathrm{LO}_{\mathrm{OPE}}$ " and "NLO $\mathrm{OPE}$ ", respectively. In order to carry out the comparison in a somewhat realistic setting, we introduce a phenomenological average momentum through

$$
k_{\mathrm{av}}^{2}(M) \equiv \frac{\int_{0}^{\infty} \mathrm{d} k k^{4} \exp \left(-\frac{\sqrt{k^{2}+M^{2}}}{T}\right)}{\int_{0}^{\infty} \mathrm{d} k k^{2} \exp \left(-\frac{\sqrt{k^{2}+M^{2}}}{T}\right)}=\frac{3 M T K_{3}\left(\frac{M}{T}\right)}{K_{2}\left(\frac{M}{T}\right)} .
$$

This should be understood just as a rough guideline; for instance we have employed a Boltzmann weight so that the same value can be used for any statistics. In any case, as can clearly be seen in figure 8(right), the correct limits are reached for all statistics considered, if only quite deep in the non-relativistic regime.

\section{Summary and outlook}

The purpose of this paper has been to suggest a general strategy for determining 2-loop thermal spectral functions at non-zero energy and momentum in the rest frame of a heat bath. As has been demonstrated with the example of the most complicated "master" structure, the result can be reduced to a convergent 2-dimensional integral, eq. (7.9), within a domain shown in figure 6. For other master spectral functions, the domain remains the same but the "coefficient functions" change; in addition, due to less symmetry, the "weight functions" split up into a larger set of independent ones (the weight functions are defined as polynomials of the phase space distributions incorporating all the temperature dependence). ${ }^{8}$

\footnotetext{
${ }^{8}$ It should be mentioned that for some of the simpler masters it is not necessary to make use of the full formalism introduced in the present paper, however if other tricks fail one can always resort to it.
} 
The specific master spectral function studied, defined by eqs. (2.5), (3.1), is peculiar in that it vanishes in the zero-temperature limit (this comes about through a complete cancellation of real and virtual corrections, and is reproduced by our numerical results). Therefore it is natural to express it as $\rho_{\mathcal{I}_{\mathrm{j}}}=T^{2} \phi\left(k_{+} / T, k_{-} / T\right)$, where $k_{ \pm} \equiv\left(k_{0} \pm k\right) / 2$ and $\phi$ is a dimensionless function. The function $\phi$ has a finite limiting value in the non-relativistic limit $k_{+}, k_{-} \gg \pi T$, but a non-trivial structure in the relativistic regime $k_{+}, k_{-} \sim \pi T$, cf. figure 7. (In the regime $k_{-} \ll \pi T$ the naive loop expansion of thermal field theory breaks down and needs to be resummed through effective field theory techniques.)

Several extensions of the current investigation can be envisaged. The most obvious challenges are to work out similar results for the other master structures appearing in eqs. (2.4), (2.9) and then to compile results for the physical observables discussed in section 2. It might also be interesting to extend the results to a situation where some of the propagators are massive; this would be relevant for the cosmological applications reviewed in ref. [14]. (As has been demonstrated with a particular non-zero mass here, it may be possible to reduce the result to a 2-dimensional integral even in the presence of masses.) Perhaps it would be nice to understand analytically the behaviour in the regime $\mathcal{K}^{2} \ll(\pi T)^{2}$. In addition the question could be posed whether, possibly with the price of introducing one further integration variable, the final result in eq. (7.9) could be cast in a more compact and transparent form. Last but not least, the computation presented involved a fair amount of error-prone hand work, so that an independent crosscheck, perhaps involving other integration variables and/or another intermediate infrared regulator, would be more than welcome.

\section{Acknowledgments}

I am grateful to D. Bödeker and Y. Schröder for helpful discussions and suggestions. This work was partly supported by the Swiss National Science Foundation (SNF) under grant 200021-140234.

\section{References}

[1] S. Caron-Huot, Asymptotics of thermal spectral functions, Phys. Rev. D 79 (2009) 125009 [arXiv:0903.3958] [INSPIRE].

[2] P.B. Arnold, G.D. Moore and L.G. Yaffe, Photon emission from ultrarelativistic plasmas, JHEP 11 (2001) 057 [hep-ph/0109064] [INSPIRE].

[3] P.B. Arnold, G.D. Moore and L.G. Yaffe, Photon emission from quark gluon plasma: Complete leading order results, JHEP 12 (2001) 009 [hep-ph/0111107] [INSPIRE].

[4] P.B. Arnold, G.D. Moore and L.G. Yaffe, Photon and gluon emission in relativistic plasmas, JHEP 06 (2002) 030 [hep-ph/0204343] [INSPIRE].

[5] E. Braaten, R.D. Pisarski and T.-C. Yuan, Production of soft dileptons in the quark-gluon plasma, Phys. Rev. Lett. 64 (1990) 2242 [InSPIRE].

[6] P. Aurenche, F. Gelis, G.D. Moore and H. Zaraket, Landau-Pomeranchuk-Migdal resummation for dilepton production, JHEP 12 (2002) 006 [hep-ph/0211036] [INSPIRE]. 
[7] G.D. Moore and J.-M. Robert, Dileptons, spectral weights and conductivity in the quark-gluon plasma, hep-ph/0607172 [INSPIRE].

[8] D. Besak and D. Bödeker, Hard thermal loops for soft or collinear external momenta, JHEP 05 (2010) 007 [arXiv: 1002.0022] [INSPIRE].

[9] A. Anisimov, D. Besak and D. Bödeker, Thermal production of relativistic Majorana neutrinos: strong enhancement by multiple soft scattering, JCAP 03 (2011) 042 [arXiv: 1012.3784] [INSPIRE].

[10] D. Besak and D. Bödeker, Thermal production of ultrarelativistic right-handed neutrinos: complete leading-order results, JCAP 03 (2012) 029 [arXiv:1202.1288] [INSPIRE].

[11] J. Ghiglieri, J. Hong, A. Kurkela, E. Lu, G.D. Moore and D. Teaney, Next-to-leading order thermal photon production in a weakly coupled quark-gluon plasma, JHEP 05 (2013) 010 [arXiv: 1302.5970] [INSPIRE].

[12] G. Aarts and J.M. Martínez Resco, Continuum and lattice meson spectral functions at nonzero momentum and high temperature, Nucl. Phys. B 726 (2005) 93 [hep-lat/0507004] [INSPIRE].

[13] M. Fukugita and T. Yanagida, Baryogenesis Without Grand Unification, Phys. Lett. B 174 (1986) 45 [INSPIRE].

[14] L. Canetti, M. Drewes, T. Frossard and M. Shaposhnikov, Dark Matter, Baryogenesis and Neutrino Oscillations from Right Handed Neutrinos, arXiv:1208.4607 [INSPIRE].

[15] A. Salvio, P. Lodone and A. Strumia, Towards leptogenesis at NLO: the right-handed neutrino interaction rate, JHEP 08 (2011) 116 [arXiv:1106.2814] [INSPIRE].

[16] M. Laine and Y. Schröder, Thermal right-handed neutrino production rate in the non-relativistic regime, JHEP 02 (2012) 068 [arXiv:1112.1205] [INSPIRE].

[17] R. Baier, B. Pire and D. Schiff, Dilepton production at finite temperature: perturbative treatment at order $\alpha_{s}$, Phys. Rev. D 38 (1988) 2814 [INSPIRE].

[18] Y. Gabellini, T. Grandou and D. Poizat, Electron-positron annihilation in thermal QCD, Annals Phys. 202 (1990) 436 [INSPIRE].

[19] T. Altherr and P. Aurenche, Finite temperature QCD corrections to lepton pair formation in a quark-gluon plasma, Z. Phys. C 45 (1989) 99 [InSPIRE].

[20] M. Laine, A. Vuorinen and Y. Zhu, Next-to-leading order thermal spectral functions in the perturbative domain, JHEP 09 (2011) 084 [arXiv:1108.1259] [INSPIRE].

[21] Y. Zhu and A. Vuorinen, The shear channel spectral function in hot Yang-Mills theory, JHEP 03 (2013) 002 [arXiv:1212.3818] [INSPIRE].

[22] G. Cuniberti, E. De Micheli and G.A. Viano, Reconstructing the thermal Green functions at real times from those at imaginary times, Commun. Math. Phys. 216 (2001) 59 [cond-mat/0109175] [INSPIRE].

[23] Y. Burnier, M. Laine and L. Mether, A test on analytic continuation of thermal imaginary-time data, Eur. Phys. J. C 71 (2011) 1619 [arXiv:1101.5534] [INSPIRE].

[24] Y. Burnier and M. Laine, Towards flavour diffusion coefficient and electrical conductivity without ultraviolet contamination, Eur. Phys. J. C 72 (2012) 1902 [arXiv:1201.1994] [INSPIRE]. 
[25] A.K. Rajantie, Feynman diagrams to three loops in three-dimensional field theory, Nucl. Phys. B 480 (1996) 729 [Erratum ibid. B 513 (1998) 761] [hep-ph/9606216] [INSPIRE].

[26] D.J. Broadhurst, J. Fleischer and O. Tarasov, Two-loop two-point functions with masses: asymptotic expansions and Taylor series, in any dimension, Z. Phys. C 60 (1993) 287 [hep-ph/9304303] [INSPIRE].

[27] V.A. Smirnov, Analytic Tools for Feynman Integrals, Springer Verlag, Berlin Germany (2012).

[28] M. Laine, M. Vepsäläinen and A. Vuorinen, Intermediate distance correlators in hot Yang-Mills theory, JHEP 12 (2010) 078 [arXiv:1011.4439] [INSPIRE].

[29] B. Garbrecht, F. Glowna and M. Herranen, Right-handed neutrino production at finite temperature: radiative corrections, soft and collinear divergences, JHEP 04 (2013) 099 [arXiv: 1302.0743] [INSPIRE].

[30] L.D. McLerran and T. Toimela, Photon and Dilepton Emission from the quark-gluon Plasma: Some General Considerations, Phys. Rev. D 31 (1985) 545 [inSPIRE].

[31] H.A. Weldon, Reformulation of finite temperature dilepton production, Phys. Rev. D 42 (1990) 2384 [INSPIRE].

[32] C. Gale and J.I. Kapusta, Vector dominance model at finite temperature, Nucl. Phys. B 357 (1991) 65 [InSPIRE].

[33] Y. Burnier and M. Laine, Massive vector current correlator in thermal QCD, JHEP 11 (2012) 086 [arXiv:1210.1064] [INSPIRE].

[34] M. Laine, M. Vepsäläinen and A. Vuorinen, Ultraviolet asymptotics of scalar and pseudoscalar correlators in hot Yang-Mills theory, JHEP 10 (2010) 010 [arXiv:1008.3263] [INSPIRE].

[35] Y. Schröder, M. Vepsäläinen, A. Vuorinen and Y. Zhu, The Ultraviolet limit and sum rule for the shear correlator in hot Yang-Mills theory, JHEP 12 (2011) 035 [arXiv:1109.6548] [INSPIRE].

[36] M.F. Zoller and K.G. Chetyrkin, OPE of the energy-momentum tensor correlator in massless QCD, JHEP 12 (2012) 119 [arXiv:1209.1516] [INSPIRE].

[37] M. Laine, Thermal right-handed neutrino self-energy in the non-relativistic regime, arXiv: 1209.2869 [INSPIRE].

[38] H.A. Weldon, Effective Fermion Masses of Order gT in High Temperature Gauge Theories with Exact Chiral Invariance, Phys. Rev. D 26 (1982) 2789 [INSPIRE].

[39] H.A. Weldon, Simple rules for discontinuities in finite temperature field theory, Phys. Rev. D 28 (1983) 2007 [INSPIRE].

[40] Y. Burnier, M. Laine and M. Vepsäläinen, Heavy quark medium polarization at next-to-leading order, JHEP 02 (2009) 008 [arXiv:0812.2105] [INSPIRE].

[41] E. Byckling and K. Kajantie, Particle Kinematics, John Wiley, New York U.S.A. (1973). 